\title{
Transnationalism and care of migrant families during pregnancy, postpartum and early-childhood: an integrative review
}

Lisa Merry ${ }^{1 *}$, Sarah Fredsted Villadsen ${ }^{2}$, Veronik Sicard $^{3}$ and Naomie Lewis-Hibbert ${ }^{4}$

\begin{abstract}
Background: Migrant families' transnational ties (i.e., connections to their countries of origin) may contribute to their hardships and/or may be a source of resiliency. A care approach that addresses these transnational ties may foster a positive identity and give coherence to experiences. We conducted an integrative review to determine what is known about transnational ties and the care of migrant families during pregnancy, postpartum and early childhood.
\end{abstract}

Methods: We searched 15 databases to identify literature reporting on a health or social program, service, or care experience of migrant families during pregnancy up to age five in a Western country (i.e., Canada, US, Australia, New Zealand or a European country). Information regarding if and how the service/program/care considered transnational ties, and care-providers' perceptions of transnational ties, was extracted, analyzed and synthesized according to transnational 'ways of belonging' and 'ways of being'.

Results: Over 34,000 records were screened; 69 articles were included. Care, programs and services examined included prenatal interventions (a mhealth app, courses, videos, and specialized antenatal care), doula support, maternity care, support groups, primary healthcare and psycho-social early intervention and early childhood programs. The results show that transnational ties in terms of 'ways of belonging' (cultural, religious and linguistic identity) are acknowledged and addressed in care, although important gaps remain. Regarding 'ways of being', including emotional, social, and economic ties with children and other family members, receipt of advice and support from family, and use of health services abroad, there is very little evidence that these are acknowledged and addressed by care-providers. Perceptions of 'ways of belonging' appear to be mixed, with some care-providers being open to and willing to adapt care to accommodate religious, cultural and linguistic differences, while others are not. How care-providers perceive the social, emotional and economic ties and/or the use of services back home, remains relatively unknown.

Conclusion: Significant knowledge gaps remain regarding care-providers' perceptions of transnational 'ways of being' and whether and how they take them into account, which may affect their relationships with migrant families and/or the effectiveness of their interventions. Continued efforts are needed to ensure care is culturally safe for migrants.

Keywords: Transnationalism, Migration, Pregnancy, Maternity care, Early childhood, Health and social care, Parenthood

\footnotetext{
* Correspondence: lisa.merry@umontreal.ca

${ }^{1}$ Faculty of Nursing, University of Montreal, Montreal, Canada

Full list of author information is available at the end of the article
}

\section{$\triangle B M C$}

(c) The Author(s). 2020 Open Access This article is licensed under a Creative Commons Attribution 4.0 International License, which permits use, sharing, adaptation, distribution and reproduction in any medium or format, as long as you give appropriate credit to the original author(s) and the source, provide a link to the Creative Commons licence, and indicate if changes were made. The images or other third party material in this article are included in the article's Creative Commons licence, unless indicated otherwise in a credit line to the material. If material is not included in the article's Creative Commons licence and your intended use is not permitted by statutory regulation or exceeds the permitted use, you will need to obtain permission directly from the copyright holder. To view a copy of this licence, visit http://creativecommons.org/licenses/by/4.0/ The Creative Commons Public Domain Dedication waiver (http://creativecommons.org/publicdomain/zero/1.0/) applies to the data made available in this article, unless otherwise stated in a credit line to the data. 


\section{Background}

Having and raising children can be challenging, particularly during resettlement in a new country [1]. Migrant parents (e.g., immigrants, refugees) may struggle financially and encounter difficulties managing cultural differences related to childbearing and parenting, for example different approaches for disciplining and socializing children. Many families must also adjust to shifting family relationships associated with changes in the family structure and gender dynamics [2-5] and cope with the loss of social networks, discrimination and navigating new health and social systems [2, 3, 6-10]. Migrant families stay connected to their countries of origin and their transnational ties may contribute to their hardships and/ or may be a source of strength and resiliency [1].

Transnational ties include 'ways of being' as well as 'ways of belonging' [11, 12]. 'Ways of being' refers to the transnational practices and relations that are sustained (i.e., economic, social, emotional and/or civic relationships and involvement) with the home country whereas 'ways of belonging' refers to the maintenance of a transnational identity (i.e., religious, cultural/ethnic, linguistic, and/or political) [11, 12]. Transnational 'ways of being' and 'ways of belonging' can influence the childbearing and parenthood experiences in a new country in a multitude of ways. Many migrant families continue to support family via sending remittances and this may affect them financially [2, 13-16]. A number of families also parent children and care for elderly family members from a distance and this too may cause financial strain as well as psychological distress [3, 15, 17-19]. Staying connected to family and friends back home may enhance feelings of isolation and loneliness and hinder cultural adaptation, while use of health services back home may have an effect on continuity of care [20-22]. Conversely, transnational ties can serve as a resource for migrant families. Connecting and visiting the home country can help maintain language and to pass on traditions to children and serve as a source of support for parenting [15, 16, 23-26]. Bilingualism may contribute to social and economic capital; maintaining culture can promote family closeness and well-being and help resist oppression [5, 7, $24,27]$. Migrants may rely on advice from family members for staying healthy during pregnancy and postpartum, for parenting (e.g., nutrition, discipline, socialization) and for resolving family conflicts [24, 25]. Family and friends back home may also provide emotional support and childcare either for children who remained behind, or for infants/children who are sent to the home country temporarily $[15,16,28-31]$. Health services may also be accessed for reasons of cost or cultural acceptability, and work/business activities may be an additional source of income [21, 32-34]. Continued engagement civically and politically may also function as a coping strategy to deal with cultural and social losses associated with migration $[7,30]$.

In the health field there is a growing interest in transnationalism and its relevance to health and care $[35,36]$. In mental health it has also been shown that an approach in care that acknowledges and addresses transnational ties may foster a positive identity and give meaning and coherence to migrant families' experiences $[37,38]$. The early years $(0-5$ years $)$ is a time when migrant families may first come into contact with health and social services, especially around the time when a child is born. It's also an opportune moment for intervention, since pregnancy and early childhood are crucial periods for child development and it is also when parents may feel overwhelmed. During this period families may interact with a range of care-providers (e.g., nurses, doctors, social-workers, educators) in various settings such as hospitals, clinics/health centers, early childhood programs and community organizations. Some may be involved in specialized programs which aim specifically to support more vulnerable families, particularly in the context of adapting to a new culture and language [31]. General care as well as specialized programs may offer services to enhance parenting skills, foster child development, promote a healthy lifestyle, and provide social connection for families experiencing isolation [31, 3942]. In this literature review, we sought to determine what is known regarding transnational ties and the health and social care of migrant parents/families during pregnancy, postpartum and early childhood in Western, high-income countries. The following research questions were addressed: 1 - Do health and social services aimed towards promoting health and providing support to parents/families during pregnancy, postpartum and early childhood ( $0-5$ years) consider the transnational contexts and experiences of migrants? 2- How do careproviders address transnational ties in their care and interventions with migrant families during pregnancy, postpartum and early-childhood? and 3-What are these care-providers' perspectives on migrant families' transnational ties to their home country?

\section{Methods}

We conducted an integrative review [43]. This type of review allows for the inclusion of diverse literature (research and discussion papers). We searched broadly to identify papers that described either the care-giving experiences of health or social care professionals or the care-receiving experiences of migrant families during pregnancy, postpartum or early childhood in Western countries. We also included papers that generally described or reported on the development or evaluation of a health or social program/intervention, or service for migrant families during pregnancy up to age five. All 
papers were then assessed to determine if there is any evidence that health and social services supporting migrant families during pregnancy, postpartum and early childhood consider and address transnational ties, including 'ways of being' and 'ways of belonging', during care with these families. We also assessed to see what information exists on care-providers' perspectives of transnational ties.

\section{Search strategy}

Searches were conducted by VS and NLH in 15 online databases: EMBASE, Web of Science, Medline, PubMed, PsycINFO, CINAHL, Scopus, Family Studies Abstracts, Global Health, Social Sciences Abstracts, Social Work Abstracts, Campbell Collaboration, Social Services Abstracts, Sociological Abstracts, and Dissertations and Thesis. The searches in CINAHL and EMBASE excluded Medline records. We selected the databases and developed the search strategy in consultation with a university librarian. Subject headings/descriptors and keywords were identified to capture key concepts including migration (e.g., immigrant, refugee, transient, asylum, resettlement), parenthood (e.g., mother, father, child-caregiver), early childhood (e.g., child, toddler, infant, pre-schooler, newborn, early childhood, child birth, pregnancy), and health/social services (e.g., health services, health care services, social services, counseling, mental health, social support). Terms were adjusted according to the vocabulary used in each database. The searches for parenthood, early childhood, migration, and health/social services were then combined using the AND Boolean operator. Keywords were searched within titles, abstracts, and keywords of articles. To ensure the literature obtained was relevant to the current migration and health and social care context, we limited the searches to the last 15 years (January 2004 to December 2018; from January to July 2019 we set-up alerts to notify us when potentially relevant literature was published after the initial searches were conducted). We also applied a language restriction (English and French) based on our language capacities. An example of a detailed database search strategy (Web of Science) is shown in Table 1.

In addition to the database searches we also handsearched the reference lists of included papers. The same time and language limitations were applied.

\section{Inclusion and exclusion criteria}

Table 2 provides a summary of the inclusion and exclusion criteria. Peer-reviewed publications including research (qualitative, quantitative, or mixed methods) and discussion papers, were considered. We also considered dissertations, however if results were published in an article, we only kept the latter. Literature reviews, study protocols, commentaries and books were excluded.
Table 1 Web of Science database search strategy

1 mother* OR father* OR parent* OR grandparent* OR grandmother* OR grandfather* OR fami** OR sibling* OR household* OR "in-law*" OR caregiver* OR (child NEAR/3 caregiver*) OR "child-caregiver" OR dad* OR mom* OR pregnan* OR childrearing OR "child rearing"

2 child* OR infant* OR newborn* OR baby OR babies OR "preschooler*" OR pregnan* OR offspring* OR toddler OR kid

3 Immigra* OR migra* OR emigra* OR refugee* OR asylum OR transient* OR undocumented OR resett ${ }^{*}$ OR settl* OR transmigration OR reestablish* OR relocate* OR exodus OR expatri* OR displac* OR exile OR deserter* OR deport* OR transnational

4 ((Social OR support) NEAR/4 service*) OR ((social OR emotional OR psycho*) NEAR/4 support) OR counsel\$̧ing OR nurs* OR intervention* OR "wellness program" OR "support services" OR therap* OR emergenc* OR ((intensive OR critical OR primary OR mental) NEAR/4 care) OR preventi* OR health* OR clinic* OR psycho* OR "caregiver NEAR/3 support"

$5 \quad 1$ and 2 and 3 and 4

6 Limit 5 to January 2004-Current

7 Limit 6 to English or French

Table 2 Inclusion and exclusion criteria

Inclusion Criteria

1. Peer reviewed publications and dissertations

2. Reported on a health or social program/intervention or service, or described the care-giving or care-receiving experiences during pregnancy, postpartum and/or early childhood

3. Examined or discussed migrant parents' (mothers, fathers, and extended family) care experiences and/or implications for the care of migrant parents/child caregivers

4. The receiving country was Canada, United States, Australia or a European country

Exclusion Criteria

1. Literature reviews, study protocols, commentaries and books

2. Dissertations if published in articles (only the published articles were retained)

3. The care/service/program/intervention targeted only one aspect of health (e.g., specialized programs for breastfeeding, nutrition, or dental care) or was not aimed at promoting health and/or it focused on families with parents or children who were ill or who had disabilities

4. The child ages were not specified or results or discussion points on early childhood were not reported/discussed separately from those of other child age groups

5. The focus was second or other generation migrants or mixed families (family members with migrant and non-migrant statuses)

6. The focus was medical tourists (i.e., those who came to the receiving country only for care)

7. Migrant parents' experiences/implications for care of migrant parents/ child caregivers were not reported or discussed separately from nonmigrants

8. Reported or described health or parenthood outcomes or experiences without any mention of health or social care experiences/interactions or implications for care of migrant parents 
Literature was included if it reported on a health or social program, intervention, or service, or if it described the care-giving experiences of health or social care professionals or if it examined the care-receiving experiences of families during pregnancy, postpartum or early childhood in Canada, the US, Australia, New Zealand or European countries. The care/service/program/intervention had to involve general health promotion activities; to keep the results manageable, literature focusing on care/services/programs/interventions that targeted only one aspect of health (e.g., breastfeeding, nutrition, dental care) were excluded. Papers must have included and examined or discussed migrant parents' (mothers, fathers, and extended family involved in parenting) care experiences and/or described or discussed implications for the care of migrant parents/child caregivers (i.e., if the focus was only on children's experiences or outcomes, the paper was excluded). Literature that examined care and services targeting families with parents or children who were ill or who had disabilities, or that focused on childhood without specifying child ages, or that did not report results for, or discuss pregnancy, postpartum or early childhood specifically, were excluded. "Migrant" was defined as anyone born outside of the country (including migrants without status); migration could have been for any reason (e.g., forced, economic) and could have been temporary or permanent [44]. If both migrants and non-migrants were included in a study or discussion paper, we retained the article only if migrant parents' experiences and/or implications for care of migrant parents/child-caregivers were reported and discussed separately from those of non-migrants.

All citations were downloaded and managed using Mendeley Desktop software (version 1.19.4). VS and NLH conducted the screening of all titles and abstracts to select potentially eligible literature. Full papers were then retrieved and reviewed to confirm eligibility. If eligibility was uncertain, a decision was made via group discussion. LM reviewed and confirmed the final selection of the included literature.

\section{Data extraction, analysis and synthesis}

For all eligible literature, we extracted and entered data into an Excel database. Data extracted included basic descriptors: the year, language and type of publication (original research, discussion paper, dissertation); the location of the study/discussion (Canada, US, Australia, New Zealand and/or European countries), information about the type of service/program/intervention and/or care given/received, the objective of the paper, and if applicable, the research design, methods, and population studied (parents and/or care-providers). For studies that examined parents, we noted who was included (mothers and/or fathers), their migration status, countries/regions of origin, length of time in the country, and/or their ethnicity. To address the research questions, information regarding if and how the service/program/care/intervention addressed transnationalism (ways of being and/or ways of belonging), as well care-providers' perspectives on transnational ties, were also extracted. We also noted any general reference made to transnational ties in the article even if it was not in relation to the intervention/ service/program/care. LM, with the support of a research assistant, assessed the methodological quality of empirical studies (qualitative, quantitative and mixed methods) using the Mixed Methods Appraisal Tool (MMAT) [45]. The MMAT uses a checklist (Yes/No/ can't tell) format with five questions that vary depending on the type of research being assessed. For qualitative research, studies were appraised on the appropriateness of the methodology and data collection methods, the adequacy of the data and its interpretation and the coherence between the data collection, analyses and findings. Quantitative studies were evaluated on the appropriateness of the sampling, randomization (if relevant), measurement and analyses, and the risk for bias. Mixed methods studies were assessed for the suitability of using a mixed methods design for the research questions being addressed, the methodological quality of each component, and the appropriateness of the 'mixing' of the qualitative and quantitative aspects of the study. No studies were excluded based on the quality assessment. All information gathered from the literature was synthesized into summary tables and text.

\section{Results}

The database searches resulted in over 93,000 records (see Additional file 1 for the search results for each database). Once duplicates were removed and the initial screening was conducted, 318 records were considered for inclusion. Thirteen articles could not be located and 236 were excluded following reading of the full text. The main reasons for exclusion at this stage were: 1 - the child ages were not specified and/or results or discussion points were reported for all child age groups together; 2migrant parents' maternity and parenthood experiences were described generally without any reference to health or social care experiences/interactions or implications for care; and 3- results and implications for migrants and non-migrants were not reported separately. Sixtynine articles were included in the review; The PRISMA flow diagram is presented in Fig. 1 and a summary of the included literature is reported in Table 3.

Almost all of the papers were published in English, only two were published in French [22,69] and just over $40 \%(n=28)$ were published within the last 5 years (2015-2019). Three papers were discussion papers [57, 76, 99], while the remaining were studies, mostly 


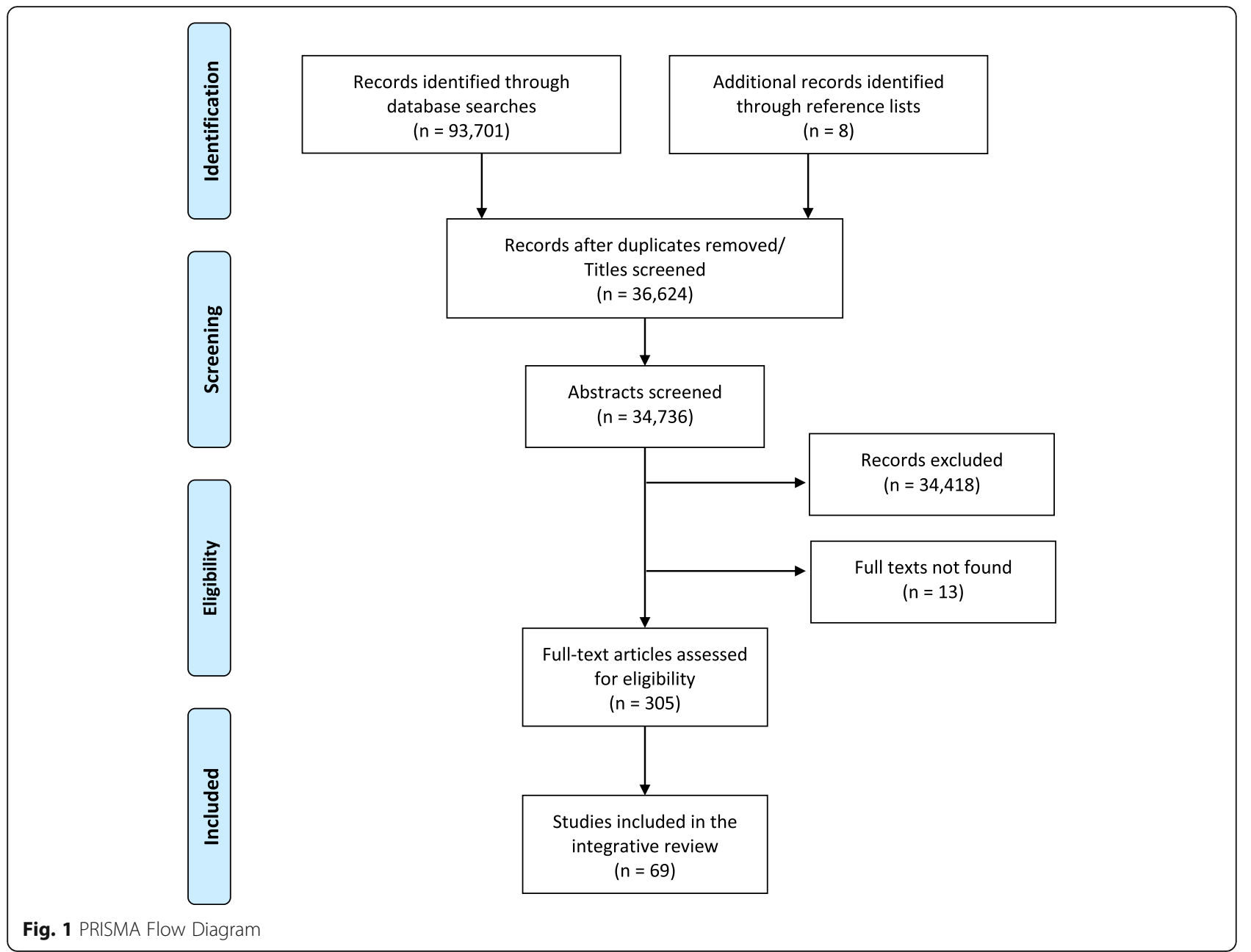

qualitative research $(n=57)$; five of these were dissertations $[52,80,82,95,105]$. Sixteen papers were discussions or studies conducted in the United States, while 13 papers were focused in Australia, followed by 11 in Canada. Papers from Europe reflected a range of countries, including Sweden (5 papers), Finland and the UK (4 papers respectively), and Germany and Ireland (3 papers respectively). (see Table 4).

The majority of the literature $(n=48 ; 70 \%)$ discussed or examined maternity care, services, or interventions (see Table 4). Most of these articles $(n=38)$ generally described experiences in receiving or giving care during pregnancy, childbirth and/or postpartum; 21 of these included only mothers $[21,50,51,55,59,61,63,64,66$, $67,71,72,74,82,89,93,95-97,106,107]$, one focused only on fathers [85], and another included both parents' experiences [109]. Eight other articles described the perspectives of healthcare providers, primarily obstetricians, nurses and midwives [53, 58, 65, 77, 78, 84, 87, 104], while six other papers described both migrant mothers' and healthcare providers' general experiences [56, 69, 70, $83,88,94]$ (see Table 3 for details).
Eleven papers focused on a specific maternity service or intervention (see Tables 3 and 4). Ten of these were directed towards migrant mothers including doula support [46, 47], a mobile health application to enhance pregnancy well-being for women from the Caribbean (by providing health information and social support) [48, 49], a nurse-practitioner led prenatal program for migrant farm-workers [57], a support group to enhance prenatal and postnatal education [41], a prenatal training course [75], a community-based antenatal service [86], a group pregnancy care initiative for Karen women from Burma [92] and a specialized antenatal clinic for refugee women [100]. One paper examined a culturally tailored prenatal health education video series that was developed for both mothers and fathers from Somalia [68].

Sixteen papers (23\% of the literature) focused on care, an intervention or a program during early-childhood (see Tables 3 and 4). These included: studies that examined migrant mothers' general experiences of accessing primary healthcare $[52,62,79]$ or early-childhood programs [105] for their children; one study that explored nurses' experiences in providing primary healthcare to 
Table 3 Description of included literature

\begin{tabular}{|c|c|c|c|c|c|c|c|}
\hline \# & $\begin{array}{l}\text { 1st Author } \\
\text { year }\end{array}$ & Objective & $\begin{array}{l}\text { Country } \\
\text { Location }\end{array}$ & $\begin{array}{l}\text { Care, program or } \\
\text { intervention } \\
\text { examined }\end{array}$ & $\begin{array}{l}\text { Design/ } \\
\text { Methodology }\end{array}$ & $\begin{array}{l}\text { Sample \& Data } \\
\text { collection methods }\end{array}$ & $\begin{array}{l}\text { Migrant groups } \\
\text { included in the }_{\text {research }}{ }^{\text {b }}\end{array}$ \\
\hline \multirow[t]{4}{*}{1.} & \multirow{4}{*}{$\begin{array}{l}\text { Akhavan } 2012 \\
\text { [46] }\end{array}$} & \multirow{4}{*}{$\begin{array}{l}\text { To explore the } \\
\text { experiences of doula } \\
\text { support among } \\
\text { foreign-born women in } \\
\text { Sweden in the context } \\
\text { of a "Community-Based } \\
\text { Doula" intervention } \\
\text { project }\end{array}$} & \multirow{4}{*}{ Sweden } & \multirow{4}{*}{$\begin{array}{l}\text { Community-based } \\
\text { doula }\end{array}$} & \multirow{4}{*}{$\begin{array}{l}\text { Qualitative } \\
\text { descriptive }\end{array}$} & 10 mothers & \multirow{3}{*}{ 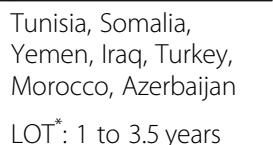 } \\
\hline & & & & & & Interviews & \\
\hline & & & & & & & \\
\hline & & & & & & & $\begin{array}{l}\text { Refugee, family } \\
\text { sponsored }\end{array}$ \\
\hline \multirow[t]{2}{*}{2.} & \multirow{2}{*}{$\begin{array}{l}\text { Akhavan } 2012 \\
\text { [47] }\end{array}$} & \multirow{2}{*}{$\begin{array}{l}\text { To describe and } \\
\text { analyze midwives' } \\
\text { experiences of doula } \\
\text { support for immigrant } \\
\text { women in Sweden }\end{array}$} & \multirow[t]{2}{*}{ Sweden } & \multirow{2}{*}{$\begin{array}{l}\text { Community-based } \\
\text { doula }\end{array}$} & \multirow{2}{*}{$\begin{array}{l}\text { Qualitative } \\
\text { descriptive }\end{array}$} & 10 midwives & \multirow[t]{2}{*}{ N/A } \\
\hline & & & & & & Interviews & \\
\hline \multirow[t]{2}{*}{3.} & \multirow{2}{*}{$\begin{array}{l}\text { AlJaberi } 2018 \\
\text { [48] }\end{array}$} & \multirow[b]{2}{*}{$\begin{array}{l}\text { To develop a } \\
\text { comprehensive } \\
\text { understanding of the } \\
\text { pregnancy health and } \\
\text { social needs of low- } \\
\text { income Caribbean im- } \\
\text { migrants towards } \\
\text { informing the develop- } \\
\text { ment of a mHealth } \\
\text { intervention }\end{array}$} & \multirow{2}{*}{$\begin{array}{l}\text { United } \\
\text { States }\end{array}$} & \multirow{2}{*}{$\begin{array}{l}\text { Pregnancy mobile } \\
\text { health intervention }\end{array}$} & \multirow{2}{*}{$\begin{array}{l}\text { Qualitative } \\
\text { descriptive }\end{array}$} & 12 mothers & Caribbean \\
\hline & & & & & & Focus groups & Immigrant \\
\hline \multirow[t]{2}{*}{4.} & \multirow{2}{*}{$\begin{array}{l}\text { AlJaberi } 2018 \\
\text { [49] }\end{array}$} & \multirow[b]{2}{*}{$\begin{array}{l}\text { To understand the } \\
\text { emotional, physical, } \\
\text { information and social } \\
\text { challenges affecting } \\
\text { low-income women's } \\
\text { prenatal well-being } \\
\text { practices towards de- } \\
\text { veloping a mHealth } \\
\text { intervention for these } \\
\text { women }\end{array}$} & \multirow{2}{*}{$\begin{array}{l}\text { United } \\
\text { States }\end{array}$} & \multirow{2}{*}{$\begin{array}{l}\text { Pregnancy mobile } \\
\text { health intervention }\end{array}$} & \multirow{2}{*}{$\begin{array}{l}\text { Qualitative } \\
\text { descriptive }\end{array}$} & 12 mothers & Caribbean \\
\hline & & & & & & Focus groups & Immigrant \\
\hline \multirow[t]{4}{*}{5.} & \multirow{4}{*}{$\begin{array}{l}\text { Almeida } 2014 \\
\text { [50] }\end{array}$} & \multirow{4}{*}{$\begin{array}{l}\text { To verify whether there } \\
\text { are differences } \\
\text { regarding women's } \\
\text { perceptions of quality } \\
\text { and appropriateness of } \\
\text { care received between } \\
\text { immigrant and native } \\
\text { women during } \\
\text { pregnancy and } \\
\text { postpartum }\end{array}$} & \multirow[t]{4}{*}{ Portugal } & \multirow{4}{*}{$\begin{array}{l}\text { Healthcare during } \\
\text { pregnancy and } \\
\text { postpartum }\end{array}$} & \multirow{4}{*}{$\begin{array}{l}\text { Qualitative } \\
\text { descriptive }\end{array}$} & 31 mothers & \multirow{2}{*}{$\begin{array}{l}\text { African countries } \\
\text { (Portuguese-speaking), } \\
\text { Brazil, Eastern } \\
\text { European countries }\end{array}$} \\
\hline & & & & & & Interviews & \\
\hline & & & & & & & LOT: 2 to 20 years \\
\hline & & & & & & & $\begin{array}{l}\text { Immigrant, } \\
\text { undocumented }\end{array}$ \\
\hline \multirow[t]{4}{*}{6.} & Almeida 2014 & To provide qualitative & Portugal & Care during & Qualitative & 31 mothers & African countries \\
\hline & & $\begin{array}{l}\text { Information on the } \\
\text { access, use and } \\
\text { perceived quality of }\end{array}$ & & $\begin{array}{l}\text { pregnancy and early } \\
\text { motherhood }\end{array}$ & descriptive & Interviews & $\begin{array}{l}\text { (Portuguese-speaking), } \\
\text { Brazil, Eastern } \\
\text { European countries }\end{array}$ \\
\hline & & $\begin{array}{l}\text { care during pregnancy } \\
\text { and early motherhood, }\end{array}$ & & & & & LOT: 2 to 20 years \\
\hline & & $\begin{array}{l}\text { reported by a group of } \\
\text { immigrants in a large } \\
\text { urban area in northern } \\
\text { Portugal; Portuguese } \\
\text { women were also } \\
\text { interviewed for } \\
\text { comparison }\end{array}$ & & & & & $\begin{array}{l}\text { Immigrant, } \\
\text { undocumented }\end{array}$ \\
\hline 7. & $\begin{array}{l}\text { Anderson } \\
2014[52]\end{array}$ & $\begin{array}{l}\text { To explore recent } \\
\text { immigrant mothers' }\end{array}$ & Canada & $\begin{array}{l}\text { Primary healthcare } \\
\text { for children } 1 \text { to } 5\end{array}$ & $\begin{array}{l}\text { Qualitative } \\
\text { descriptive }\end{array}$ & 32 mothers & $\begin{array}{l}\text { Latin American, Sri } \\
\text { Lankan Tamil }\end{array}$ \\
\hline & & $\begin{array}{l}\text { experiences accessing } \\
\text { and utilizing primary }\end{array}$ & & years old & & Interviews & LOT: $\leq 5$ years \\
\hline & & $\begin{array}{l}\text { healthcare for their } \\
\text { young children 1-5 } \\
\text { years old }\end{array}$ & & & & & $\begin{array}{l}\text { Arrived as refugee } \\
\text { claimant or was family- } \\
\text { sponsored }\end{array}$ \\
\hline
\end{tabular}


Table 3 Description of included literature (Continued)

\begin{tabular}{|c|c|c|c|c|c|c|c|}
\hline \# & $\begin{array}{l}\text { 1st Author } \\
\text { year }\end{array}$ & Objective & $\begin{array}{l}\text { Country } \\
\text { Location }\end{array}$ & $\begin{array}{l}\text { Care, program or } \\
\text { intervention } \\
\text { examined }\end{array}$ & $\begin{array}{l}\text { Design/ } \\
\text { Methodology }\end{array}$ & $\begin{array}{l}\text { Sample \& Data } \\
\text { collection methods }\end{array}$ & $\begin{array}{l}\text { Migrant groups } \\
\text { included in the }_{\text {research }}\end{array}$ \\
\hline \multirow[t]{2}{*}{8.} & \multirow[t]{2}{*}{$\begin{array}{l}\text { Ayers } 2018 \\
{[53]}\end{array}$} & \multirow{2}{*}{$\begin{array}{l}\text { To explore maternal } \\
\text { health care provider's } \\
\text { perspective of barriers } \\
\text { in providing care to } \\
\text { Marshallese women } \\
\text { and providers } \\
\text { perceived barriers of } \\
\text { access to care among } \\
\text { Marshallese women }\end{array}$} & \multirow[t]{2}{*}{$\begin{array}{l}\text { United } \\
\text { States }\end{array}$} & \multirow[t]{2}{*}{ Maternal healthcare } & \multirow[t]{2}{*}{ Phenomenology } & $\begin{array}{l}15 \text { nurses, } 2 \\
\text { obstetricians and } 2 \\
\text { other healthcare } \\
\text { providers (unspecified) }\end{array}$ & \multirow[t]{2}{*}{$\mathrm{N} / \mathrm{A}$} \\
\hline & & & & & & $\begin{array}{l}\text { Focus groups and } \\
\text { interviews }\end{array}$ & \\
\hline \multirow[t]{4}{*}{9.} & \multirow{4}{*}{$\begin{array}{l}\text { Aubé } 2019 \\
\text { [31] }\end{array}$} & \multirow{4}{*}{$\begin{array}{l}\text { To describe the } \\
\text { challenges and } \\
\text { protective factors that } \\
\text { affect the well-being of } \\
\text { migrant mothers and } \\
\text { how La Maison Bleue, } \\
\text { a community-based } \\
\text { perinatal health and } \\
\text { social centre, } \\
\text { strengthens resilience } \\
\text { among these families }\end{array}$} & \multirow[t]{4}{*}{ Canada } & \multirow{4}{*}{$\begin{array}{l}\text { A community-based } \\
\text { perinatal health and } \\
\text { social centre }\end{array}$} & \multirow{4}{*}{$\begin{array}{l}\text { Focused } \\
\text { ethnography }\end{array}$} & 24 mothers & \multirow[b]{2}{*}{$\begin{array}{l}\text { Bangladesh, Saint- } \\
\text { Lucia, Democratic Re- } \\
\text { public of Congo, } \\
\text { Morocco, Mexico, } \\
\text { Cameroon, Eritrea, } \\
\text { Pakistan, Sri Lanka, } \\
\text { India, Algeria }\end{array}$} \\
\hline & & & & & & \multirow[t]{3}{*}{$\begin{array}{l}\text { Interviews and } \\
\text { observations }\end{array}$} & \\
\hline & & & & & & & LOT: < 1 to 11 years \\
\hline & & & & & & & $\begin{array}{l}\text { Immigrant, refugee, } \\
\text { asylum seeker, } \\
\text { undocumented }\end{array}$ \\
\hline \multirow[t]{2}{*}{10.} & \multirow[t]{2}{*}{$\begin{array}{l}\text { Balaam } 2016 \\
{[54]}\end{array}$} & \multirow{2}{*}{$\begin{array}{l}\text { To explore the } \\
\text { experience of voluntary } \\
\text { and non-statutory sec- } \\
\text { tor workers supporting } \\
\text { asylum-seeking and } \\
\text { refugee women during } \\
\text { pregnancy and early } \\
\text { motherhood }\end{array}$} & \multirow[t]{2}{*}{$\begin{array}{l}\text { United } \\
\text { Kingdom }\end{array}$} & \multirow{2}{*}{$\begin{array}{l}\text { Voluntary and non- } \\
\text { statutory support } \\
\text { for childbearing } \\
\text { refugee and asylum } \\
\text { seeking women }\end{array}$} & \multirow[t]{2}{*}{$\begin{array}{l}\text { Qualitative } \\
\text { descriptive }\end{array}$} & $\begin{array}{l}19 \text { volunteer and non- } \\
\text { statutory (paid and un- } \\
\text { paid) workers }\end{array}$ & \multirow[t]{2}{*}{ N/A } \\
\hline & & & & & & $\begin{array}{l}\text { Individual and focus } \\
\text { group interviews }\end{array}$ & \\
\hline \multirow{3}{*}{11.} & \multirow{3}{*}{$\begin{array}{l}\text { Barkensjo } \\
2018[55]\end{array}$} & \multirow{3}{*}{$\begin{array}{l}\text { To describe women's } \\
\text { experiences of clinical } \\
\text { encounters throughout } \\
\text { pregnancy and } \\
\text { childbirth, when living } \\
\text { as undocumented } \\
\text { migrants in Sweden }\end{array}$} & \multirow{3}{*}{ Sweden } & \multirow[t]{3}{*}{ Maternity care } & \multirow{3}{*}{$\begin{array}{l}\text { Qualitative } \\
\text { descriptive }\end{array}$} & 13 mothers & \multirow{2}{*}{$\begin{array}{l}\text { Macedonia, Romania, } \\
\text { Bosnia, Albania, } \\
\text { Somalia, Afghanistan, } \\
\text { Serbia, Chechnya, } \\
\text { Morocco, Kosovo }\end{array}$} \\
\hline & & & & & & Interviews & \\
\hline & & & & & & & $\begin{array}{l}\text { Undocumented, EU } \\
\text { citizens without } \\
\text { residency permits }\end{array}$ \\
\hline \multirow[t]{3}{*}{12.} & \multirow[t]{3}{*}{$\begin{array}{l}\text { Barona-Vilar } \\
2013[56]\end{array}$} & \multirow{3}{*}{$\begin{array}{l}\text { To explore the } \\
\text { experiences and } \\
\text { perceptions of } \\
\text { parenthood and } \\
\text { maternal health care } \\
\text { among Latin American } \\
\text { women living in Spain }\end{array}$} & \multirow[t]{3}{*}{ Spain } & \multirow[t]{3}{*}{ Maternal health care } & \multirow[t]{3}{*}{$\begin{array}{l}\text { Qualitative } \\
\text { descriptive }\end{array}$} & $\begin{array}{l}26 \text { mothers and } 24 \\
\text { midwives }\end{array}$ & Bolivia, Ecuador \\
\hline & & & & & & Focus groups & LOT: 1 to 9 years \\
\hline & & & & & & & $\begin{array}{l}\text { Immigrant, } \\
\text { undocumented }\end{array}$ \\
\hline \multirow[t]{4}{*}{13.} & $\begin{array}{l}\text { Beaudet } 2016 \\
{[22]}\end{array}$ & $\begin{array}{l}\text { To evaluate a support- } \\
\text { group intervention de- } \\
\text { veloped collaboratively } \\
\text { between a community } \\
\text { organization and a } \\
\text { local health clinic to }\end{array}$ & Canada & $\begin{array}{l}\text { Community } \\
\text { support-group inter- } \\
\text { vention for immi- } \\
\text { grant mothers }\end{array}$ & $\begin{array}{l}\text { Qualitative } \\
\text { descriptive }\end{array}$ & $\begin{array}{l}13 \text { mothers, } 2 \\
\text { administrators, } 2 \\
\text { support group workers } \\
\& 1 \text { social worker } \\
\text { (support group } \\
\text { committee), Mothers }\end{array}$ & $\begin{array}{l}\text { China, Colombia, } \\
\text { Korea, Egypt, France, } \\
\text { Honduras, India, Iran, } \\
\text { Iraq, Japan, Lebanon, } \\
\text { Libya, Poland, } \\
\text { Singapore, Taiwan }\end{array}$ \\
\hline & & $\begin{array}{l}\text { address isolation and } \\
\text { support recently- } \\
\text { arrived immigrant }\end{array}$ & & & & $\begin{array}{l}\text { and children } \\
\text { participating in the } \\
\text { support group, and }\end{array}$ & $\begin{array}{l}\text { LOT: } 8 \text { women < } 2 \\
\text { years, others longer }\end{array}$ \\
\hline & & $\begin{array}{l}\text { mothers with children } \\
\text { aged } 0 \text { to } 2 \text { years }\end{array}$ & & & & $\begin{array}{l}\text { Administrators \& } \\
\text { workers from the } \\
\text { community } \\
\text { organization }\end{array}$ & Immigrant \\
\hline & & & & & & $\begin{array}{l}\text { Interviews, discussion } \\
\text { groups, observations } \\
\text { of support group } \\
\text { sessions and } \\
\text { committee meetings, }\end{array}$ & \\
\hline
\end{tabular}


Table 3 Description of included literature (Continued)

\begin{tabular}{|c|c|c|c|c|c|c|c|}
\hline \# & $\begin{array}{l}\text { 1st Author } \\
\text { year }\end{array}$ & Objective & $\begin{array}{l}\text { Country } \\
\text { Location }\end{array}$ & $\begin{array}{l}\text { Care, program or } \\
\text { intervention } \\
\text { examined }\end{array}$ & $\begin{array}{l}\text { Design/ } \\
\text { Methodology }^{a}\end{array}$ & $\begin{array}{l}\text { Sample \& Data } \\
\text { collection methods }\end{array}$ & $\begin{array}{l}\text { Migrant groups } \\
\text { included in the } \\
\text { research }^{b}\end{array}$ \\
\hline \multirow[b]{2}{*}{14.} & \multirow[b]{2}{*}{$\begin{array}{l}\text { Bircher } 2009 \\
\text { [57] }\end{array}$} & \multirow[b]{2}{*}{$\begin{array}{l}\text { To describe the } \\
\text { challenges of migrant } \\
\text { farm workers during } \\
\text { pregnancy and to } \\
\text { suggest ways that } \\
\text { advanced practice } \\
\text { nurses can provide } \\
\text { cost effective, } \\
\text { competent professional } \\
\text { care to reduce or } \\
\text { eliminate the obstacles } \\
\text { to prenatal care for this } \\
\text { population }\end{array}$} & \multirow[b]{2}{*}{$\begin{array}{l}\text { United } \\
\text { States }\end{array}$} & \multirow[b]{2}{*}{$\begin{array}{l}\text { Prenatal care } \\
\text { offered by a nurse } \\
\text { practitioner }\end{array}$} & \multirow[b]{2}{*}{ Discussion paper } & $\begin{array}{l}\text { and a review of } \\
\text { documents (support- } \\
\text { group participation } \\
\text { logbook, child } \\
\text { assessments, support- } \\
\text { group journal) }\end{array}$ & \\
\hline & & & & & & & \\
\hline \multirow[t]{2}{*}{15.} & \multirow[t]{2}{*}{$\begin{array}{l}\text { Boerleider } \\
2014 \text { [58] }\end{array}$} & \multirow{2}{*}{$\begin{array}{l}\text { To gain insight into } \\
\text { how Dutch postnatal } \\
\text { care providers - } \\
\text { maternity care } \\
\text { assistants -address } \\
\text { issues encountered } \\
\text { when providing care } \\
\text { for non-western } \\
\text { women }\end{array}$} & \multirow[t]{2}{*}{ Netherlands } & \multirow[t]{2}{*}{ Postnatal care } & \multirow[t]{2}{*}{$\begin{array}{l}\text { Qualitative } \\
\text { descriptive }\end{array}$} & $\begin{array}{l}15 \text { maternity-care } \\
\text { assistants }\end{array}$ & \multirow[t]{2}{*}{ N/A } \\
\hline & & & & & & Interviews & \\
\hline \multirow[t]{3}{*}{16.} & \multirow[t]{3}{*}{$\begin{array}{l}\text { Briscoe } 2009 \\
{[59]}\end{array}$} & \multirow{3}{*}{$\begin{array}{l}\text { To explore the } \\
\text { experience of } \\
\text { maternity care by } \\
\text { asylum seekers and } \\
\text { one refugee }\end{array}$} & \multirow[t]{3}{*}{$\begin{array}{l}\text { United } \\
\text { Kingdom }\end{array}$} & \multirow[t]{3}{*}{ Maternity care } & \multirow[t]{3}{*}{$\begin{array}{l}\text { Multiple case } \\
\text { study }\end{array}$} & 4 mothers & $\begin{array}{l}\text { Afghanistan, Congo, } \\
\text { Rwanda, Somalia }\end{array}$ \\
\hline & & & & & & $\begin{array}{l}\text { Photographs, } \\
\text { observations, and }\end{array}$ & $\begin{array}{l}\text { LOT: < } 1 \text { year to just } \\
\text { under } 3 \text { years }\end{array}$ \\
\hline & & & & & & interviews & Refugee, asylum seeker \\
\hline \multirow[t]{3}{*}{17.} & \multirow[t]{3}{*}{$\begin{array}{l}\text { Busch } 2018 \\
\text { [60] }\end{array}$} & \multirow{3}{*}{$\begin{array}{l}\text { To investigate } \\
\text { challenges and } \\
\text { possible solutions in a } \\
\text { specialized early } \\
\text { childhood education } \\
\text { and care (ECEC) } \\
\text { program for refugee } \\
\text { children }\end{array}$} & \multirow[t]{3}{*}{ Germany } & \multirow[t]{3}{*}{$\begin{array}{l}\text { A specialized ECEC } \\
\text { for refugee children }\end{array}$} & \multirow{3}{*}{$\begin{array}{l}\text { Mixed-methods } \\
\text { (qualitative } \\
\text { descriptive } \\
\text { followed by a } \\
\text { survey based on } \\
\text { the qualitative } \\
\text { data) }\end{array}$} & $\begin{array}{l}28 \text { early-childhood } \\
\text { educators }\end{array}$ & \multirow[t]{3}{*}{ N/A } \\
\hline & & & & & & $\begin{array}{l}96 \text { early-childhood ed- } \\
\text { ucators (a second } \\
\text { sample) }\end{array}$ & \\
\hline & & & & & & $\begin{array}{l}\text { Interviews and } \\
\text { questionnaire }\end{array}$ & \\
\hline \multirow[t]{4}{*}{18.} & \multirow[t]{4}{*}{$\begin{array}{l}\text { Carolan } 2010 \\
\text { [61] }\end{array}$} & \multirow{4}{*}{$\begin{array}{l}\text { To explore the } \\
\text { experiences and } \\
\text { concerns of an African- } \\
\text { born sample of preg- } \\
\text { nant women receiving } \\
\text { antenatal care in } \\
\text { Melbourne }\end{array}$} & \multirow[t]{4}{*}{ Australia } & \multirow[t]{4}{*}{ Antenatal care } & \multirow[t]{4}{*}{$\begin{array}{l}\text { Qualitative } \\
\text { descriptive }\end{array}$} & 18 mothers & $\begin{array}{l}\text { Ethiopia, Sudan, Eritrea, } \\
\text { Kenya, Somalia }\end{array}$ \\
\hline & & & & & & Interviews & $\begin{array}{l}\text { LOT: < } 1 \text { year to } 2 \\
\text { years }\end{array}$ \\
\hline & & & & & & & $\begin{array}{l}\text { Refugee, family- } \\
\text { reunification visa, } \\
\text { immigrant }\end{array}$ \\
\hline & & & & & & & $\begin{array}{l}\text { Dinka, Amharic, } \\
\text { Christian }\end{array}$ \\
\hline \multirow[t]{2}{*}{19.} & \multirow[t]{2}{*}{ Clark 2007 [62] } & \multirow[b]{2}{*}{$\begin{array}{l}\text { To identify Mexican } \\
\text { American mother's } \\
\text { expectations from } \\
\text { children's health care } \\
\text { services (during the } \\
\text { first } 19 \text { months of their } \\
\text { child's life) }\end{array}$} & \multirow{2}{*}{$\begin{array}{l}\text { United } \\
\text { States }\end{array}$} & Children's health & Focused & 28 mothers & Mexico \\
\hline & & & & & ethnography & Interviews & $\begin{array}{l}\text { Immigrant, } \\
\text { undocumented }\end{array}$ \\
\hline 20. & $\begin{array}{l}\text { Coley } 2012 \\
{[41]}\end{array}$ & $\begin{array}{l}\text { To 1) describe the } \\
\text { development of the }\end{array}$ & $\begin{array}{l}\text { United } \\
\text { States }\end{array}$ & $\begin{array}{l}\text { Support group to } \\
\text { enhance prenatal }\end{array}$ & $\begin{array}{l}\text { Qualitative } \\
\text { descriptive }\end{array}$ & 7 mothers & $\begin{array}{l}\text { Nigeria, Ghana, Nepal, } \\
\text { Mexico, India, Jordan }\end{array}$ \\
\hline
\end{tabular}


Table 3 Description of included literature (Continued)

\begin{tabular}{|c|c|c|c|c|c|c|c|}
\hline \# & $\begin{array}{l}\text { 1st Author } \\
\text { year }\end{array}$ & Objective & $\begin{array}{l}\text { Country } \\
\text { Location }\end{array}$ & $\begin{array}{l}\text { Care, program or } \\
\text { intervention } \\
\text { examined }\end{array}$ & $\begin{array}{l}\text { Design/ } \\
\text { Methodology }\end{array}$ & $\begin{array}{l}\text { Sample \& Data } \\
\text { collection methods }\end{array}$ & $\begin{array}{l}\text { Migrant groups } \\
\text { included in the } \\
\text { research }^{b}\end{array}$ \\
\hline & & $\begin{array}{l}\text { Moms Matter support } \\
\text { group; 2) illustrate the } \\
\text { effects of incorporating } \\
\text { cultural competence } \\
\text { and social support in } \\
\text { childbirth education; } \\
\text { and 3) suggest } \\
\text { implications for the } \\
\text { future development of } \\
\text { pregnancy support } \\
\text { programs for diverse } \\
\text { immigrant populations }\end{array}$ & & $\begin{array}{l}\text { and postnatal } \\
\text { education for } \\
\text { immigrant mothers }\end{array}$ & & & Immigrant \\
\hline \multirow[t]{3}{*}{21.} & \multirow{3}{*}{$\begin{array}{l}\text { Coutinho } 2014 \\
\text { [63] }\end{array}$} & \multirow{3}{*}{$\begin{array}{l}\text { To identify the unmet } \\
\text { expectations of } \\
\text { Portuguese immigrant } \\
\text { women, for the } \\
\text { National Health } \\
\text { System, during } \\
\text { pregnancy, childbirth } \\
\text { and postpartum }\end{array}$} & \multirow[t]{3}{*}{ Portugal } & \multirow[t]{3}{*}{ Maternity care } & \multirow{3}{*}{$\begin{array}{l}\text { Qualitative } \\
\text { descriptive }\end{array}$} & 82 mothers & \multirow{2}{*}{$\begin{array}{l}\text { Brazil, Ukraine, China, } \\
\text { Moldova, Russia, } \\
\text { France }\end{array}$} \\
\hline & & & & & & Interviews & \\
\hline & & & & & & & Immigrant \\
\hline \multirow{3}{*}{22.} & \multirow{3}{*}{$\begin{array}{l}\text { Doering } 2015 \\
\text { [64] }\end{array}$} & \multirow{3}{*}{$\begin{array}{l}\text { To explore how some } \\
\text { Japanese women } \\
\text { experienced } \\
\text { pregnancy, labor and } \\
\text { birth care in New } \\
\text { Zealand }\end{array}$} & \multirow{3}{*}{$\begin{array}{l}\text { New } \\
\text { Zealand }\end{array}$} & \multirow[t]{3}{*}{ Maternity care } & \multirow{3}{*}{$\begin{array}{l}\text { Qualitative } \\
\text { descriptive }\end{array}$} & 13 mothers & Japan \\
\hline & & & & & & Interviews and a focus & LOT: 2 to 19 years \\
\hline & & & & & & & Immigrant \\
\hline \multirow[t]{2}{*}{23.} & \multirow[t]{2}{*}{$\begin{array}{l}\text { Degni } 2012 \\
{[65]}\end{array}$} & \multirow{2}{*}{$\begin{array}{l}\text { To explore physicians } \\
\text { and nurses/midwives } \\
\text { communication when } \\
\text { providing reproductive } \\
\text { and maternity health } \\
\text { care to Somali women } \\
\text { in Finland }\end{array}$} & \multirow[t]{2}{*}{ Finland } & \multirow[t]{2}{*}{ Maternity care } & \multirow[t]{2}{*}{$\begin{array}{l}\text { Qualitative } \\
\text { description }\end{array}$} & $\begin{array}{l}10 \text { obstetricians, } 7 \\
\text { nurses, and } 8 \\
\text { midwives }\end{array}$ & \multirow[t]{2}{*}{ N/A } \\
\hline & & & & & & $\begin{array}{l}\text { Individual and focus } \\
\text { group interviews }\end{array}$ & \\
\hline \multirow[t]{3}{*}{24.} & \multirow{3}{*}{$\begin{array}{l}\text { Degni } 2014 \\
{[66]}\end{array}$} & \multirow{3}{*}{$\begin{array}{l}\text { To explore immigrant } \\
\text { Somali women's } \\
\text { experiences of } \\
\text { reproductive and } \\
\text { maternity health care } \\
\text { services and their } \\
\text { perceptions of the } \\
\text { service providers }\end{array}$} & \multirow[t]{3}{*}{ Finland } & \multirow[t]{3}{*}{ Maternity care } & \multirow{3}{*}{$\begin{array}{l}\text { Qualitative } \\
\text { descriptive }\end{array}$} & 70 mothers & Somalia \\
\hline & & & & & & Focus groups & $\begin{array}{l}\text { LOT: 'recently } \\
\text { migrated' }\end{array}$ \\
\hline & & & & & & & Refugee \\
\hline \multirow[t]{3}{*}{25.} & \multirow[t]{3}{*}{$\begin{array}{l}\text { Dempsey } 2016 \\
\text { [67] }\end{array}$} & \multirow{3}{*}{$\begin{array}{l}\text { To explore migrant } \\
\text { Eastern European } \\
\text { women's experience of } \\
\text { pregnancy in Ireland }\end{array}$} & \multirow[t]{3}{*}{ Ireland } & \multirow[t]{3}{*}{ Maternity care } & \multirow[t]{3}{*}{$\begin{array}{l}\text { Grounded } \\
\text { theory approach }\end{array}$} & 12 mothers & $\begin{array}{l}\text { Poland, Lithuania, } \\
\text { Hungary, Czech } \\
\text { Republic }\end{array}$ \\
\hline & & & & & & Interviews & LOT: 1 to 8 years \\
\hline & & & & & & & Economic immigrant \\
\hline \multirow[t]{3}{*}{26.} & \multirow{3}{*}{$\begin{array}{l}\text { DeStephano } \\
2010[68]\end{array}$} & \multirow{3}{*}{$\begin{array}{l}\text { To determine the } \\
\text { acceptability of a } \\
\text { culturally tailored } \\
\text { prenatal health } \\
\text { education video series } \\
\text { for Somali women and } \\
\text { explore health } \\
\text { providers' perceptions } \\
\text { regarding usefulness of } \\
\text { the videos in } \\
\text { facilitating improved } \\
\text { client-provider } \\
\text { communication }\end{array}$} & United & Culturally tailored & Quantitative & 22 mothers, 2 fathers & Somalia \\
\hline & & & & $\begin{array}{l}\text { prenatal health } \\
\text { education video } \\
\text { series for Somali }\end{array}$ & $\begin{array}{l}\text { descriptive with } \\
\text { a qualitative } \\
\text { component }\end{array}$ & $\begin{array}{l}\text { and obstetricians who } \\
\text { cared for the } 22 \\
\text { women }\end{array}$ & Refugee \\
\hline & & & & women & & Questionnaires & \\
\hline 27. & $\begin{array}{l}\text { Gabai } 2013 \\
\text { [69] }\end{array}$ & $\begin{array}{l}\text { To explore the } \\
\text { experiences of patients }\end{array}$ & France & Maternity care & $\begin{array}{l}\text { Grounded } \\
\text { theory }\end{array}$ & $\begin{array}{l}4 \text { mothers, } 10 \\
\text { obstetricians and }\end{array}$ & $\begin{array}{l}\text { Lebanon, Ivory Coast, } \\
\text { Democratic Republic of }\end{array}$ \\
\hline
\end{tabular}


Table 3 Description of included literature (Continued)

\begin{tabular}{|c|c|c|c|c|c|c|c|}
\hline \# & $\begin{array}{l}1 \text { st Author } \\
\text { year }\end{array}$ & Objective & $\begin{array}{l}\text { Country } \\
\text { Location }\end{array}$ & $\begin{array}{l}\text { Care, program or } \\
\text { intervention } \\
\text { examined }\end{array}$ & $\begin{array}{l}\text { Design/ } \\
\text { Methodology }^{\mathrm{a}}\end{array}$ & $\begin{array}{l}\text { Sample \& Data } \\
\text { collection methods }\end{array}$ & $\begin{array}{l}\text { Migrant groups } \\
\text { included in the } \\
\text { research }\end{array}$ \\
\hline & & and maternity care- & & & & midwives & Congo, Tunisia \\
\hline & & $\begin{array}{l}\text { givers in a maternity } \\
\text { context }\end{array}$ & & & & Interviews & LOT: 3 to 11 years \\
\hline & & & & & & & Immigrant \\
\hline \multirow[t]{4}{*}{28.} & \multirow[t]{4}{*}{$\begin{array}{l}\text { Grewal } 2008 \\
\text { [70] }\end{array}$} & \multirow{4}{*}{$\begin{array}{l}\text { To describe new } \\
\text { immigrant Punjabi } \\
\text { women's perinatal } \\
\text { experiences and the } \\
\text { ways that traditional } \\
\text { beliefs and practices } \\
\text { are legitimized and } \\
\text { incorporated into the } \\
\text { Canadian health care } \\
\text { context }\end{array}$} & \multirow[t]{4}{*}{ Canada } & \multirow[t]{4}{*}{ Perinatal care } & \multirow[t]{4}{*}{$\begin{array}{l}\text { Qualitative } \\
\text { descriptive }\end{array}$} & $\begin{array}{l}15 \text { mothers, } 5 \text { public } \\
\text { health nurses }\end{array}$ & India \\
\hline & & & & & & \multirow{3}{*}{$\begin{array}{l}\text { Interviews and a focus } \\
\text { group }\end{array}$} & LOT: $\leq 5$ years \\
\hline & & & & & & & Immigrant \\
\hline & & & & & & & Punjabi \\
\hline \multirow[t]{3}{*}{29.} & \multirow{3}{*}{$\begin{array}{l}\text { Higginbottom } \\
2013 \text { [71] }\end{array}$} & \multirow{3}{*}{$\begin{array}{l}\text { To map out the } \\
\text { experiences of } \\
\text { immigrant Sudanese } \\
\text { women in maternity } \\
\text { services }\end{array}$} & \multirow[t]{3}{*}{ Canada } & \multirow[t]{3}{*}{ Maternity services } & \multirow{3}{*}{$\begin{array}{l}\text { Focused } \\
\text { ethnography }\end{array}$} & 12 mothers & Sudan \\
\hline & & & & & & \multirow{2}{*}{$\begin{array}{l}\text { Focus group } \\
\text { interviews }\end{array}$} & LOT: $\leq 5$ years \\
\hline & & & & & & & Refugee \\
\hline \multirow[t]{3}{*}{30.} & \multirow[t]{3}{*}{ Hill 2012 [72] } & \multirow{3}{*}{$\begin{array}{l}\text { To describe Somali } \\
\text { immigrant women's } \\
\text { health care experiences } \\
\text { and beliefs regarding } \\
\text { pregnancy and birth }\end{array}$} & \multirow{3}{*}{$\begin{array}{l}\text { United } \\
\text { States }\end{array}$} & \multirow[t]{3}{*}{ Maternity care } & \multirow{3}{*}{$\begin{array}{l}\text { Qualitative } \\
\text { descriptive }\end{array}$} & 18 mothers & Somalia \\
\hline & & & & & & \multirow{2}{*}{$\begin{array}{l}\text { Focus group } \\
\text { interviews }\end{array}$} & LOT: 1.5 to 12 years \\
\hline & & & & & & & Refugee \\
\hline 31. & $\begin{array}{l}\text { Hurley } 2014 \\
{[73]}\end{array}$ & $\begin{array}{l}\text { To investigate the } \\
\text { challenges and } \\
\text { innovative practices in } \\
\text { early childhood special } \\
\text { education (ECSE) } \\
\text { services for preschool } \\
\text { aged children who are } \\
\text { refugees }\end{array}$ & $\begin{array}{l}\text { United } \\
\text { States }\end{array}$ & $\begin{array}{l}\text { Early childhood } \\
\text { special education } \\
\text { (ECSE) services }\end{array}$ & $\begin{array}{l}\text { Qualitative } \\
\text { descriptive }\end{array}$ & $\begin{array}{l}28 \text { early-childhood } \\
\text { educators } \\
\text { Interviews }\end{array}$ & $\mathrm{N} / \mathrm{A}$ \\
\hline 32. & Iliadi 2008 [74] & $\begin{array}{l}\text { To examine whether } \\
\text { refugee women } \\
\text { resettled in Greece, } \\
\text { receive antenatal care } \\
\text { and to explore possible } \\
\text { factors that may } \\
\text { influence their attitude } \\
\text { towards maternal care }\end{array}$ & Greece & Maternity care & $\begin{array}{l}\text { Focused } \\
\text { ethnography }\end{array}$ & $\begin{array}{l}26 \text { mothers } \\
\text { Interviews }\end{array}$ & $\begin{array}{l}\text { Iraq, Iran, Sudan, } \\
\text { Lebanon, Syria, } \\
\text { Afghanistan, Armenia, } \\
\text { Turkey, Albania, Serbia, } \\
\text { Zaire } \\
\text { Refugee }\end{array}$ \\
\hline \multirow[t]{3}{*}{33.} & \multirow{3}{*}{$\begin{array}{l}\text { Karl-Trummer } \\
2006[75]\end{array}$} & \multirow{3}{*}{$\begin{array}{l}\text { To evaluate a prenatal } \\
\text { training course } \\
\text { developed for } \\
\text { pregnant migrant/ } \\
\text { ethnic women }\end{array}$} & Italy & \multirow{3}{*}{$\begin{array}{l}\text { Prenatal training } \\
\text { course for migrant } \\
\text { women }\end{array}$} & \multirow{3}{*}{$\begin{array}{l}\text { Mixed-methods } \\
\text { (qualitative } \\
\text { descriptive in } \\
\text { conjunction } \\
\text { with a survey) }\end{array}$} & \multirow{3}{*}{$\begin{array}{l}41 \text { mothers and } 32 \\
\text { healthcare providers } \\
\text { Interviews and } \\
\text { questionnaire }\end{array}$} & Turkey, India, Pakistan \\
\hline & & & Austria & & & & Immigrant \\
\hline & & & & & & & \\
\hline \multirow{2}{*}{34.} & \multirow{2}{*}{$\begin{array}{l}\text { Lebiger-Vogel } \\
2019[76]\end{array}$} & \multirow[b]{2}{*}{$\begin{array}{l}\text { To present and discuss } \\
\text { the FIRST STEPS project } \\
\text { in Belgium and the } \\
\text { FIRST STEPS project in } \\
\text { Frankfurt and Berlin } \\
\text { (FIRST STEPS is a } \\
\text { prevention/support } \\
\text { intervention offered to } \\
\text { immigrant women in } \\
\text { early childhood and } \\
\text { which aims to optimize } \\
\text { the early } \\
\text { developmental } \\
\text { environment of } \\
\text { children) }\end{array}$} & Belgium & \multirow{2}{*}{$\begin{array}{l}\text { Early-childhood } \\
\text { support program for } \\
\text { immigrant parents }\end{array}$} & \multirow{2}{*}{ Discussion paper } & & \\
\hline & & & Germany & & & & \\
\hline 35. & $\begin{array}{l}\text { Lyberg } 2012 \\
\text { [77] }\end{array}$ & $\begin{array}{l}\text { To illuminate midwives' } \\
\text { and public health }\end{array}$ & Norway & Maternity care & $\begin{array}{l}\text { Qualitative } \\
\text { descriptive }\end{array}$ & $\begin{array}{l}5 \text { midwives and } 1 \\
\text { public health nurse }\end{array}$ & N/A \\
\hline
\end{tabular}


Table 3 Description of included literature (Continued)

\begin{tabular}{|c|c|c|c|c|c|c|c|}
\hline \# & $\begin{array}{l}\text { 1st Author } \\
\text { year }\end{array}$ & Objective & $\begin{array}{l}\text { Country } \\
\text { Location }\end{array}$ & $\begin{array}{l}\text { Care, program or } \\
\text { intervention } \\
\text { examined }\end{array}$ & $\begin{array}{l}\text { Design/ } \\
\text { Methodology }\end{array}$ & $\begin{array}{l}\text { Sample \& Data } \\
\text { collection methods }\end{array}$ & $\begin{array}{l}\text { Migrant groups } \\
\text { included in the } \\
\text { research }^{\text {b }}\end{array}$ \\
\hline & & $\begin{array}{l}\text { nurses' perceptions of } \\
\text { managing and } \\
\text { supporting prenatal } \\
\text { and postnatal migrant } \\
\text { women in Norway }\end{array}$ & & & & Focus groups & \\
\hline \multirow[t]{2}{*}{36.} & \multirow[t]{2}{*}{$\begin{array}{l}\text { Lyons } 2008 \\
\text { [78] }\end{array}$} & \multirow{2}{*}{$\begin{array}{l}\text { To explore the } \\
\text { experiences, } \\
\text { understanding and } \\
\text { perspectives of } \\
\text { maternity service } \\
\text { providers when } \\
\text { working with ethnic } \\
\text { minority women in } \\
\text { Dublin maternity } \\
\text { services during } 2002 \\
\text { and } 2003\end{array}$} & \multirow[t]{2}{*}{ Ireland } & \multirow[t]{2}{*}{ Maternity services } & \multirow[t]{2}{*}{$\begin{array}{l}\text { Grounded } \\
\text { theory approach }\end{array}$} & $\begin{array}{l}42 \text { obstetricians, } \\
\text { midwives, nurses, and } \\
\text { key informants from } \\
\text { specialized areas of } \\
\text { infection control, social } \\
\text { services and } \\
\text { bereavement services }\end{array}$ & \multirow[t]{2}{*}{ N/A } \\
\hline & & & & & & $\begin{array}{l}\text { Focus groups and } \\
\text { interviews }\end{array}$ & \\
\hline \multirow[t]{4}{*}{37.} & \multirow[t]{4}{*}{$\begin{array}{l}\text { Mangrio } 2017 \\
\text { [79] }\end{array}$} & \multirow{4}{*}{$\begin{array}{l}\text { To shed light on the } \\
\text { experience of non- } \\
\text { European immigrants } \\
\text { with Sweden's child } \\
\text { health care system }\end{array}$} & \multirow[t]{4}{*}{ Sweden } & \multirow[t]{4}{*}{ Child health care } & \multirow[t]{4}{*}{$\begin{array}{l}\text { Qualitative } \\
\text { descriptive }\end{array}$} & $\begin{array}{l}14 \text { mothers and } 5 \\
\text { fathers }\end{array}$ & \multirow{2}{*}{$\begin{array}{l}\text { Afghanistan, Chile, } \\
\text { India, Iraq, Kurdistan, } \\
\text { Kuwait, Lebanon, } \\
\text { Pakistan, Palestine, } \\
\text { Venezuela, Vietnam }\end{array}$} \\
\hline & & & & & & \multirow[t]{3}{*}{ Interviews } & \\
\hline & & & & & & & LOT: 2 to 22 years \\
\hline & & & & & & & Immigrant, refugee \\
\hline 38. & $\begin{array}{l}\text { McLaughlin } \\
2012 \text { [80] }\end{array}$ & $\begin{array}{l}\text { To explore the lived } \\
\text { experiences of } \\
\text { parenting amongst a } \\
\text { group of Burmese } \\
\text { refugee mothers and } \\
\text { their perceptions of } \\
\text { how facilitated } \\
\text { playgroups assist them } \\
\text { in their parenting role }\end{array}$ & Australia & $\begin{array}{l}\text { Facilitated } \\
\text { playgroup }\end{array}$ & Phenomenology & $\begin{array}{l}9 \text { mothers, } 2 \\
\text { playgroup staff and } 1 \\
\text { kindergarten teacher } \\
\text { Focus groups, } \\
\text { interviews }\end{array}$ & $\begin{array}{l}\text { Burma } \\
\text { Refugee }\end{array}$ \\
\hline \multirow[t]{3}{*}{39.} & \multirow[t]{3}{*}{$\begin{array}{l}\text { Merry } 2011 \\
{[81]}\end{array}$} & \multirow{3}{*}{$\begin{array}{l}\text { To gain a greater } \\
\text { understanding of the } \\
\text { barriers asylum seeking } \\
\text { women face in } \\
\text { accessing health and } \\
\text { social services } \\
\text { postpartum }\end{array}$} & \multirow[t]{3}{*}{ Canada } & \multirow[t]{3}{*}{$\begin{array}{l}\text { Health and social } \\
\text { services postpartum }\end{array}$} & \multirow[t]{3}{*}{$\begin{array}{l}\text { Qualitative } \\
\text { descriptive }\end{array}$} & 112 mothers & $\begin{array}{l}\text { Africa, Asia, Europe, } \\
\text { Latin America }\end{array}$ \\
\hline & & & & & & \multirow{2}{*}{$\begin{array}{l}\text { Review of nurses' } \\
\text { notes }\end{array}$} & LOT: $\leq 5$ years \\
\hline & & & & & & & Asylum seeker \\
\hline \multirow[t]{3}{*}{40.} & \multirow{3}{*}{$\begin{array}{l}\text { Mukasa } 2016 \\
\text { [82] }\end{array}$} & \multirow[b]{3}{*}{$\begin{array}{l}\text { To 1) understand the } \\
\text { disparities in access to } \\
\text { maternal and child } \\
\text { health (MCH) services } \\
\text { experienced by recent } \\
\text { African immigrant } \\
\text { mothers in the United } \\
\text { States; 2) explore } \\
\text { circumstances that led } \\
\text { to MCH access } \\
\text { disparities experienced } \\
\text { by this population; and } \\
\text { 3) understand how } \\
\text { access disparities } \\
\text { affected participants' } \\
\text { overall experience of } \\
\text { seeking MCH care } \\
\text { services }\end{array}$} & \multirow{3}{*}{$\begin{array}{l}\text { United } \\
\text { States }\end{array}$} & \multirow{3}{*}{$\begin{array}{l}\text { Maternal and child } \\
\text { health services }\end{array}$} & \multirow[t]{3}{*}{ Phenomenology } & 11 mothers & Sub-Saharan Africa \\
\hline & & & & & & Interviews & LOT: 1.5 to 4 years \\
\hline & & & & & & & $\begin{array}{l}\text { Immigrant, refugee, } \\
\text { asylum seeker }\end{array}$ \\
\hline \multirow[t]{2}{*}{41.} & \multirow[t]{2}{*}{$\begin{array}{l}\text { Nabb } 2006 \\
{[83]}\end{array}$} & \multirow{2}{*}{$\begin{array}{l}\text { To explore the } \\
\text { perceptions of } \\
\text { pregnant asylum- } \\
\text { seekers in relation to } \\
\text { the provision of }\end{array}$} & \multirow[t]{2}{*}{$\begin{array}{l}\text { United } \\
\text { Kingdom }\end{array}$} & \multirow[t]{2}{*}{ Maternity care } & \multirow[t]{2}{*}{$\begin{array}{l}\text { Qualitative } \\
\text { descriptive }\end{array}$} & \multirow{2}{*}{$\begin{array}{l}10 \text { mothers and } 5 \\
\text { healthcare } \\
\text { professionals } \\
\text { Interviews }\end{array}$} & $\begin{array}{l}\text { Africa, Asia, Eastern } \\
\text { Europe }\end{array}$ \\
\hline & & & & & & & Asylum seeker \\
\hline
\end{tabular}


Table 3 Description of included literature (Continued)

\begin{tabular}{|c|c|c|c|c|c|c|c|}
\hline$\#$ & $\begin{array}{l}\text { 1st Author } \\
\text { year }\end{array}$ & Objective & $\begin{array}{l}\text { Country } \\
\text { Location }\end{array}$ & $\begin{array}{l}\text { Care, program or } \\
\text { intervention } \\
\text { examined }\end{array}$ & $\begin{array}{l}\text { Design/ } \\
\text { Methodology }^{\text {a }}\end{array}$ & $\begin{array}{l}\text { Sample \& Data } \\
\text { collection methods }\end{array}$ & $\begin{array}{l}\text { Migrant groups } \\
\text { included in the } \\
\text { research }^{\text {b }}\end{array}$ \\
\hline & & $\begin{array}{l}\text { maternity care while in } \\
\text { emergency accommo- } \\
\text { dation in the UK }\end{array}$ & & & & & \\
\hline \multirow[t]{2}{*}{42.} & \multirow[t]{2}{*}{ Ng 2011 [84] } & \multirow{2}{*}{$\begin{array}{l}\text { To understand the } \\
\text { difficulties health care } \\
\text { professionals face } \\
\text { when delivering } \\
\text { prenatal care to } \\
\text { immigrant women }\end{array}$} & \multirow[t]{2}{*}{ Canada } & \multirow[t]{2}{*}{ Prenatal care } & \multirow[t]{2}{*}{$\begin{array}{l}\text { Qualitative } \\
\text { descriptive }\end{array}$} & $\begin{array}{l}3 \text { midwives, } 5 \text { nurses } \\
\text { practitioners, and } 2 \\
\text { obstetricians }\end{array}$ & \multirow[t]{2}{*}{ N/A } \\
\hline & & & & & & Interviews & \\
\hline \multirow[t]{3}{*}{43.} & \multirow[t]{3}{*}{ Ny 2006 [85] } & \multirow{3}{*}{$\begin{array}{l}\text { To describe how men } \\
\text { from the Middle East } \\
\text { experience Swedish } \\
\text { maternity and child } \\
\text { health care }\end{array}$} & \multirow[t]{3}{*}{ Sweden } & \multirow{3}{*}{$\begin{array}{l}\text { Maternal and child } \\
\text { healthcare }\end{array}$} & \multirow{3}{*}{$\begin{array}{l}\text { Qualitative } \\
\text { descriptive }\end{array}$} & 16 fathers & \multirow{3}{*}{$\begin{array}{l}\text { Middle-East } \\
\text { LOT: 1-15 years } \\
\text { Immigrant }\end{array}$} \\
\hline & & & & & & Interviews and focus & \\
\hline & & & & & & groups & \\
\hline \multirow{5}{*}{44.} & \multirow{5}{*}{$\begin{array}{l}\text { Owens } 2016 \\
\text { [86] }\end{array}$} & \multirow{5}{*}{$\begin{array}{l}\text { To explore the } \\
\text { perceptions of care } \\
\text { experienced by } \\
\text { refugees and migrant } \\
\text { women of culturally } \\
\text { and linguistically } \\
\text { diverse backgrounds } \\
\text { who had participated } \\
\text { in a community-based } \\
\text { antenatal service spe- } \\
\text { cializing in maternity } \\
\text { care for multicultural } \\
\text { women }\end{array}$} & \multirow[t]{5}{*}{ Australia } & \multirow{5}{*}{$\begin{array}{l}\text { Community-based } \\
\text { antenatal service } \\
\text { specializing in } \\
\text { maternity care of } \\
\text { women from } \\
\text { culturally and } \\
\text { linguistically diverse } \\
\text { backgrounds }\end{array}$} & \multirow[t]{5}{*}{ Phenomenology } & 12 mothers & \multirow{2}{*}{$\begin{array}{l}\text { Indonesia, Pakistan } \\
\text { Vietnam, Iran, Sudan, } \\
\text { Burma, Thailand }\end{array}$} \\
\hline & & & & & & Interviews & \\
\hline & & & & & & & LOT: 1-10 years \\
\hline & & & & & & & Immigrant, refugee \\
\hline & & & & & & & $\begin{array}{l}\text { Bhatak, Baloch, } \\
\text { Catholic, Muslim, Bari, } \\
\text { Chin, Karen }\end{array}$ \\
\hline \multirow[t]{2}{*}{45.} & \multirow[t]{2}{*}{$\begin{array}{l}\text { Pelaez } 2017 \\
\text { [87] }\end{array}$} & \multirow{2}{*}{$\begin{array}{l}\text { To explore health care } \\
\text { professionals' } \\
\text { perspectives of } \\
\text { challenges newly- } \\
\text { arrived migrant women } \\
\text { to Canada coming } \\
\text { from non-western } \\
\text { countries face when } \\
\text { needing maternity care } \\
\text { in order to better } \\
\text { understand clinical } \\
\text { practices towards these } \\
\text { women }\end{array}$} & \multirow[t]{2}{*}{ Canada } & \multirow[t]{2}{*}{ Maternity care } & \multirow[t]{2}{*}{$\begin{array}{l}\text { Multiple case } \\
\text { study }\end{array}$} & $\begin{array}{l}3 \text { family physicians, } 5 \\
\text { obstetricians, } 4 \\
\text { medical residents, } 1 \\
\text { nutritionist, } 1 \\
\text { anesthesiologist, } 7 \\
\text { social workers, } 1 \text { art } \\
\text { therapist, } 1 \\
\text { psychologist, } 1 \\
\text { spiritual consultant, } \\
\text { and } 39 \text { nurses }\end{array}$ & \multirow[t]{2}{*}{$\mathrm{N} / \mathrm{A}$} \\
\hline & & & & & & Interviews & \\
\hline \multirow[t]{4}{*}{46.} & \multirow[t]{4}{*}{$\begin{array}{l}\text { Phillimore } \\
2016 \text { [88] }\end{array}$} & \multirow{4}{*}{$\begin{array}{l}\text { To examine the } \\
\text { reasons why migrants' } \\
\text { access to antenatal } \\
\text { care is poor }\end{array}$} & \multirow[t]{4}{*}{$\begin{array}{l}\text { United } \\
\text { Kingdom }\end{array}$} & \multirow[t]{4}{*}{ Antenatal care } & $\begin{array}{l}\text { Mixed-methods } \\
\text { (Qualitative } \\
\text { descriptive in }\end{array}$ & $\begin{array}{l}82 \text { mothers and } 18 \\
\text { community health } \\
\text { staff, general }\end{array}$ & $\begin{array}{l}28 \text { countries including } \\
\text { China, Iran, Pakistan, } \\
\text { Poland, Zimbabwe }\end{array}$ \\
\hline & & & & & $\begin{array}{l}\text { conjunction } \\
\text { with a }\end{array}$ & $\begin{array}{l}\text { practitioners, } \\
\text { pregnancy outreach }\end{array}$ & LOT: $\leq 5$ years \\
\hline & & & & & & $\begin{array}{l}\text { workers, hospital staff } \\
\text { and third sector } \\
\text { workers }\end{array}$ & $\begin{array}{l}\text { Immigrant, refugee, } \\
\text { asylum seeker, } \\
\text { undocumented }\end{array}$ \\
\hline & & & & & & $\begin{array}{l}\text { Questionnaire and } \\
\text { interviews }\end{array}$ & \\
\hline 47. & Qureshi 2013 & To describe the & United & Maternity care & Ethnography & 26 mothers & Pakistan \\
\hline & & $\begin{array}{l}\text { comparative birthing } \\
\text { experiences of } \\
\text { Pakistani immigrant }\end{array}$ & & & & Interviews & $\begin{array}{l}\text { LOT: average of } 12 \\
\text { years }\end{array}$ \\
\hline & & $\begin{array}{l}\text { women in Pakistan and } \\
\text { the United States }\end{array}$ & & & & & Immigrant \\
\hline 48. & Renzaho 2014 & To explore the views & Australia & Pregnancy and & Qualitative & 35 mothers & Afghanistan, Africa, \\
\hline & & $\begin{array}{l}\text { and perceptions of } \\
\text { migrant women in } \\
\text { Dandenong, Australia, }\end{array}$ & & postnatal care & descriptive & Focus groups & $\begin{array}{l}\text { China, Palestine, } \\
\text { Lebanon, Syria, Iran, } \\
\text { Jordan }\end{array}$ \\
\hline & & $\begin{array}{l}\text { about sociocultural } \\
\text { barriers and health }\end{array}$ & & & & & LOT: 2 to 11 years \\
\hline
\end{tabular}


Table 3 Description of included literature (Continued)

\begin{tabular}{|c|c|c|c|c|c|c|c|}
\hline$\#$ & $\begin{array}{l}\text { 1st Author } \\
\text { year }\end{array}$ & Objective & $\begin{array}{l}\text { Country } \\
\text { Location }\end{array}$ & $\begin{array}{l}\text { Care, program or } \\
\text { intervention } \\
\text { examined }\end{array}$ & $\begin{array}{l}\text { Design/ } \\
\text { Methodology }^{\text {a }}\end{array}$ & $\begin{array}{l}\text { Sample \& Data } \\
\text { collection methods }\end{array}$ & $\begin{array}{l}\text { Migrant groups } \\
\text { included in the } \\
\text { research }^{\text {b }}\end{array}$ \\
\hline & & $\begin{array}{l}\text { needs during } \\
\text { pregnancy and in the } \\
\text { postnatal period }\end{array}$ & & & & & Immigrant \\
\hline \multirow{4}{*}{49.} & \multirow{4}{*}{$\begin{array}{l}\text { Rickmeyer } \\
2015 \text { [90] }\end{array}$} & \multirow{4}{*}{$\begin{array}{l}\text { To present preliminary } \\
\text { results from a project } \\
\text { that aims to evaluate } \\
\text { the FIRST STEPS } \\
\text { program, which is an } \\
\text { early-childhood parent- } \\
\text { ing support and child } \\
\text { development interven- } \\
\text { tion; preliminary results } \\
\text { included attendance } \\
\text { rates to the program, } \\
\text { socio-demographics of } \\
\text { the participating popu- } \\
\text { lation and vignettes to } \\
\text { illustrate some of the } \\
\text { positive effects for } \\
\text { families }\end{array}$} & \multirow[t]{4}{*}{ Germany } & \multirow{4}{*}{$\begin{array}{l}\text { Early-childhood } \\
\text { support program for } \\
\text { immigrant parents }\end{array}$} & \multirow{4}{*}{$\begin{array}{l}\text { Randomized } \\
\text { control trial } \\
\text { (with a } \\
\text { qualitative } \\
\text { component) }\end{array}$} & 224 mothers & \multirow[b]{2}{*}{$\begin{array}{l}\text { Ethiopia, Eritrea, Kenya, } \\
\text { Sudan, Benin, } \\
\text { Democratic Republic of } \\
\text { Congo, Ghana, Nigeria, } \\
\text { Mexico, Venezuela, } \\
\text { Algeria, Egypt, } \\
\text { Morocco, Tunisia, } \\
\text { Bulgaria, Poland, } \\
\text { Romania, Croatia, } \\
\text { Kosovo, Montenegro, } \\
\text { Serbia, Turkey, } \\
\text { Afghanistan, India, } \\
\text { Pakistan, Palestine, } \\
\text { Saudi Arabia, Syria, } \\
\text { China, Japan, Korea, } \\
\text { Vietnam }\end{array}$} \\
\hline & & & & & & $\begin{array}{l}\text { Questionnaires and } \\
\text { vignettes }\end{array}$ & \\
\hline & & & & & & & LOT: $\leq 3$ years \\
\hline & & & & & & & Immigrant \\
\hline \multirow[t]{4}{*}{50.} & \multirow[t]{4}{*}{$\begin{array}{l}\text { Riggs } 2012 \\
\text { [91] }\end{array}$} & \multirow{4}{*}{$\begin{array}{l}\text { To explore experiences } \\
\text { of using maternal and } \\
\text { child health services, } \\
\text { from the perspective of } \\
\text { families from refugee } \\
\text { backgrounds and } \\
\text { service providers }\end{array}$} & \multirow[t]{4}{*}{ Australia } & \multirow[t]{4}{*}{$\begin{array}{l}\text { Maternal and child } \\
\text { health services }\end{array}$} & \multirow[t]{4}{*}{$\begin{array}{l}\text { Qualitative } \\
\text { descriptive }\end{array}$} & \multirow{3}{*}{$\begin{array}{l}87 \text { mothers and } 5 \\
\text { healthcare providers } \\
\text { (nurses, other } \\
\text { healthcare workers } \\
\text { and bicultural workers) }\end{array}$} & $\begin{array}{l}\text { Iraq, Burma, Lebanon, } \\
\text { Bhutan, Sudan }\end{array}$ \\
\hline & & & & & & & LOT: 1.5 to 8.5 years \\
\hline & & & & & & & Refugee \\
\hline & & & & & & $\begin{array}{l}\text { Focus groups and } \\
\text { interviews }\end{array}$ & $\begin{array}{l}\text { Karen, Assyrian } \\
\text { Chaldean }\end{array}$ \\
\hline \multirow[t]{4}{*}{51.} & \multirow{4}{*}{$\begin{array}{l}\text { Riggs } 2017 \\
{[92]}\end{array}$} & \multirow{4}{*}{$\begin{array}{l}\text { To describe the } \\
\text { experiences of group } \\
\text { pregnancy care for } \\
\text { Karen women from } \\
\text { Burma who have } \\
\text { resettled in Melbourne, } \\
\text { Australia }\end{array}$} & \multirow[t]{4}{*}{ Australia } & \multirow{4}{*}{$\begin{array}{l}\text { Group pregnancy } \\
\text { care }\end{array}$} & \multirow{4}{*}{$\begin{array}{l}\text { Qualitative } \\
\text { descriptive }\end{array}$} & 19 mothers & Burma \\
\hline & & & & & & Focus groups & $\begin{array}{l}\text { LOT: < } 1 \text { year to } 10 \\
\text { years }\end{array}$ \\
\hline & & & & & & & Refugee \\
\hline & & & & & & & Karen \\
\hline \multirow{3}{*}{52.} & \multirow{3}{*}{$\begin{array}{l}\text { Russo } 2015 \\
{[93]}\end{array}$} & \multirow{3}{*}{$\begin{array}{l}\text { To explore the } \\
\text { experiences of Afghan } \\
\text { women living in } \\
\text { Melbourne throughout } \\
\text { pregnancy, birth, and } \\
\text { early motherhood, and } \\
\text { gain insight into the } \\
\text { aspects of their } \\
\text { experiences that they } \\
\text { perceive as positively } \\
\text { and negatively } \\
\text { impacting their } \\
\text { emotional wellbeing }\end{array}$} & \multirow[t]{3}{*}{ Australia } & \multirow[t]{3}{*}{ Maternity care } & \multirow{3}{*}{$\begin{array}{l}\text { Qualitative } \\
\text { descriptive }\end{array}$} & 38 mothers & Afghanistan \\
\hline & & & & & & Focus groups and & LOT: 1 to 6 years \\
\hline & & & & & & & Refugee \\
\hline \multirow[t]{3}{*}{53.} & \multirow[t]{3}{*}{$\begin{array}{l}\text { Sanchez } 2017 \\
\text { [94] }\end{array}$} & \multirow{3}{*}{$\begin{array}{l}\text { To describe Mexican } \\
\text { immigrant women } \\
\text { experiences of } \\
\text { pregnancy and birth } \\
\text { and to identify the } \\
\text { approaches that } \\
\text { midwives use when } \\
\text { caring for these } \\
\text { women }\end{array}$} & \multirow[t]{3}{*}{$\begin{array}{l}\text { United } \\
\text { States }\end{array}$} & \multirow[t]{3}{*}{ Midwifery care } & \multirow[t]{3}{*}{$\begin{array}{l}\text { Qualitative } \\
\text { descriptive }\end{array}$} & $\begin{array}{l}20 \text { mothers and } 5 \\
\text { nurse-midwives }\end{array}$ & Mexico \\
\hline & & & & & & Interviews & $\begin{array}{l}\text { LOT: } 16 \text { were in US < } \\
3 \text { years, others longer }\end{array}$ \\
\hline & & & & & & & $\begin{array}{l}\text { Undocumented, } \\
\text { immigrant }\end{array}$ \\
\hline \multirow[t]{3}{*}{54.} & \multirow{3}{*}{$\begin{array}{l}\text { Schmiedigen } \\
2013 \text { [95] }\end{array}$} & To describe the & United & Maternity care & Interpretive & 8 mothers & Brazil \\
\hline & & $\begin{array}{l}\text { subjective experience } \\
\text { of Brazilian women }\end{array}$ & States & & phenomenology & Interviews & LOT: $\leq 10$ years \\
\hline & & entering motherhood & & & & & Immigrant \\
\hline
\end{tabular}


Table 3 Description of included literature (Continued)

\begin{tabular}{|c|c|c|c|c|c|c|c|}
\hline \# & $\begin{array}{l}\text { 1st Author } \\
\text { year }\end{array}$ & Objective & $\begin{array}{l}\text { Country } \\
\text { Location }\end{array}$ & $\begin{array}{l}\text { Care, program or } \\
\text { intervention } \\
\text { examined }\end{array}$ & $\begin{array}{l}\text { Design/ } \\
\text { Methodology }^{a}\end{array}$ & $\begin{array}{l}\text { Sample \& Data } \\
\text { collection methods }\end{array}$ & $\begin{array}{l}\text { Migrant groups } \\
\text { included in the } \\
\text { research }\end{array}$ \\
\hline & & in the United States & & & & & \\
\hline \multirow[t]{3}{*}{55.} & \multirow[t]{3}{*}{ Seo 2017 [96] } & \multirow{3}{*}{$\begin{array}{l}\text { To understand Korean } \\
\text { immigrant women's } \\
\text { common experiences } \\
\text { and practices of } \\
\text { utilizing health care } \\
\text { services in the United } \\
\text { States during childbirth }\end{array}$} & \multirow{3}{*}{$\begin{array}{l}\text { United } \\
\text { States }\end{array}$} & \multirow{3}{*}{$\begin{array}{l}\text { Health care services } \\
\text { during childbirth }\end{array}$} & \multirow{3}{*}{$\begin{array}{l}\text { Interpretive } \\
\text { phenomenology }\end{array}$} & 15 mothers & Korea \\
\hline & & & & & & Interviews & $\begin{array}{l}\text { LOT: } 1.5 \text { years to } 19 \\
\text { years }\end{array}$ \\
\hline & & & & & & & Immigrant \\
\hline \multirow{3}{*}{56.} & \multirow{3}{*}{$\begin{array}{l}\text { Shafiei } 2012 \\
{[97]}\end{array}$} & \multirow{3}{*}{$\begin{array}{l}\text { To explore immigrant } \\
\text { Afghan women's views } \\
\text { and experiences of } \\
\text { maternity care in } \\
\text { Melbourne, Australia }\end{array}$} & \multirow{3}{*}{ Australia } & \multirow[t]{3}{*}{ Maternity care } & \multirow{3}{*}{$\begin{array}{l}\text { Mixed-methods } \\
\text { design (survey } \\
\text { followed by } \\
\text { qualitative } \\
\text { interviews) }\end{array}$} & 40 mothers & Afghanistan \\
\hline & & & & & & $\begin{array}{l}\text { Questionnaire and } \\
\text { Interviews }\end{array}$ & $\begin{array}{l}\text { LOT: half were } \leq 5 \\
\text { years, other half }>5 \\
\text { years }\end{array}$ \\
\hline & & & & & & & Refugee \\
\hline \multirow{5}{*}{57.} & \multirow{5}{*}{$\begin{array}{l}\text { Signorelli } 2015 \\
\text { [98] }\end{array}$} & \multirow{5}{*}{$\begin{array}{l}\text { To describe the STAR } \\
\text { TTerS Early childhood } \\
\text { program (a multimodal } \\
\text { program that aims to } \\
\text { support child } \\
\text { development and } \\
\text { trauma recovery, and } \\
\text { enhance parenting } \\
\text { confidence and skills), } \\
\text { and to report the } \\
\text { results from a } \\
\text { community project } \\
\text { with Karen and } \\
\text { Mandaean refugee } \\
\text { communities which } \\
\text { aimed to better tailor } \\
\text { services for these } \\
\text { populations }\end{array}$} & \multirow[t]{5}{*}{ Australia } & \multirow{5}{*}{$\begin{array}{l}\text { Early childhood } \\
\text { program for refugee } \\
\text { families }\end{array}$} & \multirow{5}{*}{$\begin{array}{l}\text { Qualitative } \\
\text { descriptive }\end{array}$} & \multirow{4}{*}{$\begin{array}{l}48 \text { male and female } \\
\text { participants including } \\
\text { parents, grandparents, } \\
\text { other care-givers com- } \\
\text { munity leaders and } \\
\text { other community } \\
\text { members }\end{array}$} & Burma, Iraq \\
\hline & & & & & & & $\begin{array}{l}\text { LOT: very few were } \\
\text { recent arrivals }\end{array}$ \\
\hline & & & & & & & Refugee \\
\hline & & & & & & & Karen, Mandaean \\
\hline & & & & & & \multirow[t]{2}{*}{$\begin{array}{l}\text { Focus groups and } \\
\text { interviews }\end{array}$} & \\
\hline 58. & $\begin{array}{l}\text { Signorelli } 2017 \\
\text { [99] }\end{array}$ & $\begin{array}{l}\text { To explore the } \\
\text { implementation of a } \\
\text { model to address } \\
\text { access and other } \\
\text { challenges in early } \\
\text { childhood work with } \\
\text { refugee families and } \\
\text { communities, with the } \\
\text { intent to increase } \\
\text { service uptake }\end{array}$ & Australia & $\begin{array}{l}\text { Early childhood } \\
\text { program for refugee } \\
\text { families }\end{array}$ & Discussion paper & & \\
\hline \multirow[t]{2}{*}{59.} & \multirow[t]{2}{*}{$\begin{array}{l}\text { Stapleton } \\
2013[100]\end{array}$} & \multirow[t]{2}{*}{$\begin{array}{l}\text { To explore whether } \\
\text { maternity care for } \\
\text { women from refugee } \\
\text { backgrounds attending } \\
\text { a specialist antenatal } \\
\text { clinic in a tertiary } \\
\text { Australian public } \\
\text { hospital, could be } \\
\text { improved }\end{array}$} & \multirow[t]{2}{*}{ Australia } & \multirow[t]{2}{*}{$\begin{array}{l}\text { Antenatal clinic for } \\
\text { refugee women }\end{array}$} & \multirow[t]{2}{*}{$\begin{array}{l}\text { Mixed-methods } \\
\text { (retrospective } \\
\text { cohort, survey, } \\
\text { and qualitative } \\
\text { descriptive) }\end{array}$} & $\begin{array}{l}4348 \text { mothers (hospital } \\
\text { data), } 42 \text { service-users } \\
\text { (mothers), } 147 \text { hospital } \\
\text { staff, } 3 \text { clinic staff, } 3 \\
\text { hospital managers, } 2 \\
\text { interpreting coordina- } \\
\text { tors, and } 5 \text { key } \\
\text { stakeholders }\end{array}$ & \multirow[t]{2}{*}{$\begin{array}{l}\text { Africa and Middle East } \\
\text { including Somalia, } \\
\text { Sudan, Afghanistan, } \\
\text { Burundi, Liberia } \\
\text { Refugee }\end{array}$} \\
\hline & & & & & & $\begin{array}{l}\text { Surveys, interviews, } \\
\text { focus groups, hospital } \\
\text { and clinic databases } \\
\text { and chart audit }\end{array}$ & \\
\hline \multirow[t]{2}{*}{60.} & \multirow{2}{*}{$\begin{array}{l}\text { Stewart } 2015 \\
{[101]}\end{array}$} & \multirow{2}{*}{$\begin{array}{l}\text { To evaluate a social } \\
\text { support intervention } \\
\text { (support groups }\end{array}$} & \multirow[t]{2}{*}{ Canada } & \multirow{2}{*}{$\begin{array}{l}\text { Psychosocial } \\
\text { intervention for } \\
\text { refugee parents }\end{array}$} & \multirow{2}{*}{$\begin{array}{l}\text { Mixed-methods } \\
\text { (pre-test post- } \\
\text { test design and }\end{array}$} & \multirow{2}{*}{$\begin{array}{l}38 \text { mothers and } 47 \\
\text { fathers } 21 \text { peer and } \\
\text { professional mentors }\end{array}$} & Sudan, Zimbabwe \\
\hline & & & & & & & LOT: $<5$ years \\
\hline
\end{tabular}
consisting of like-ethnic and like-gender peers) for refugee new parents

refugee parents with young children (4 months to 5 years) test design and qualitative descriptive)

Interviews and questionnaires (social support, loneliness and isolation, coping, parenting stress) 
Table 3 Description of included literature (Continued)

\begin{tabular}{|c|c|c|c|c|c|c|c|}
\hline$\#$ & $\begin{array}{l}\text { 1st Author } \\
\text { year }\end{array}$ & Objective & $\begin{array}{l}\text { Country } \\
\text { Location }\end{array}$ & $\begin{array}{l}\text { Care, program or } \\
\text { intervention } \\
\text { examined }\end{array}$ & $\begin{array}{l}\text { Design/ } \\
\text { Methodology }\end{array}$ & $\begin{array}{l}\text { Sample \& Data } \\
\text { collection methods }\end{array}$ & $\begin{array}{l}\text { Migrant groups } \\
\text { included in the } \\
\text { research }^{\text {b }}\end{array}$ \\
\hline \multirow[t]{4}{*}{61.} & \multirow{4}{*}{$\begin{array}{l}\text { Stewart } 2017 \\
\text { [102] }\end{array}$} & \multirow{4}{*}{$\begin{array}{l}\text { To examine support } \\
\text { needs of African } \\
\text { refugee new parents in } \\
\text { Canada to guide } \\
\text { development of a } \\
\text { tailored support } \\
\text { intervention }\end{array}$} & \multirow[t]{4}{*}{ Canada } & \multirow{4}{*}{$\begin{array}{l}\text { Psychosocial } \\
\text { intervention for } \\
\text { refugee parents } \\
\text { with young children } \\
\text { ( } 4 \text { months to } 5 \\
\text { years) }\end{array}$} & \multirow{4}{*}{$\begin{array}{l}\text { Mixed-methods } \\
\text { (qualitative } \\
\text { descriptive and } \\
\text { questionnaires) }\end{array}$} & \multirow{3}{*}{$\begin{array}{l}29 \text { fathers and } 43 \\
\text { mothers (additional } \\
\text { parents also } \\
\text { participated in the } \\
\text { group interviews but } \\
\text { unclear how many), } \\
\text { and } 15 \text { service- } \\
\text { providers and } 15 \\
\text { policy-influences/ } \\
\text { makers }\end{array}$} & Zimbabwe, Sudan \\
\hline & & & & & & & LOT: < 5 years \\
\hline & & & & & & & Refugee, asylum seeker \\
\hline & & & & & & $\begin{array}{l}\text { Individual and group } \\
\text { interviews and } \\
\text { questionnaires (social } \\
\text { support, coping) }\end{array}$ & \\
\hline \multirow[t]{3}{*}{62.} & \multirow[t]{3}{*}{$\begin{array}{l}\text { Stewart } 2018 \\
{[103]}\end{array}$} & \multirow{3}{*}{$\begin{array}{l}\text { To develop and test an } \\
\text { accessible and } \\
\text { culturally appropriate } \\
\text { social support } \\
\text { intervention designed } \\
\text { to meet the support } \\
\text { needs and preferences } \\
\text { identified by African } \\
\text { refugee parents of } \\
\text { young children }\end{array}$} & \multirow[t]{3}{*}{ Canada } & \multirow{3}{*}{$\begin{array}{l}\text { Social support } \\
\text { intervention for } \\
\text { refugee parents } \\
\text { with young children } \\
\text { ( } 4 \text { months to } 5 \\
\text { years) }\end{array}$} & \multirow{3}{*}{$\begin{array}{l}\text { Qualitative } \\
\text { descriptive }\end{array}$} & $\begin{array}{l}47 \text { fathers and } 38 \\
\text { mothers }\end{array}$ & Sudan, Zimbabwe \\
\hline & & & & & & Individual and group & LOT: < 5 years \\
\hline & & & & & & & Refugee \\
\hline \multirow[t]{2}{*}{63.} & \multirow{2}{*}{$\begin{array}{l}\text { Tobin } 2014 \\
\text { [104] }\end{array}$} & \multirow[b]{2}{*}{$\begin{array}{l}\text { To explore midwives' } \\
\text { perceptions and } \\
\text { experiences of } \\
\text { providing care to } \\
\text { women in the asylum } \\
\text { process and to gain } \\
\text { insight into how } \\
\text { midwives can be } \\
\text { equipped and } \\
\text { supported to provide } \\
\text { more effective care to } \\
\text { this group in the future }\end{array}$} & \multirow[t]{2}{*}{ Ireland } & \multirow[t]{2}{*}{ Maternity care } & \multirow{2}{*}{$\begin{array}{l}\text { Qualitative } \\
\text { descriptive }\end{array}$} & 10 midwives & \multirow[t]{2}{*}{ N/A } \\
\hline & & & & & & Interviews & \\
\hline \multirow[t]{4}{*}{64.} & \multirow{4}{*}{$\begin{array}{l}\text { Vesely } 2011 \\
{[105]}\end{array}$} & \multirow{4}{*}{$\begin{array}{l}\text { To gain greater } \\
\text { understanding of the } \\
\text { lived experiences of } \\
\text { immigrant mothers of } \\
\text { young children as they } \\
\text { parented in the U.S. } \\
\text { and interacted with the } \\
\text { Early childhood care } \\
\text { and education system }\end{array}$} & \multirow{4}{*}{$\begin{array}{l}\text { United } \\
\text { States }\end{array}$} & \multirow{4}{*}{$\begin{array}{l}\text { Early childhood care } \\
\text { and education } \\
\text { mothers }\end{array}$} & \multirow{4}{*}{$\begin{array}{l}\text { Ethnographic } \\
\text { and grounded } \\
\text { theory } \\
\text { approaches }\end{array}$} & 41 mothers, 4 fathers & \multirow{3}{*}{$\begin{array}{l}\text { Ethiopia, El Salvador, } \\
\text { Eritrea, Sudan, Ghana, } \\
\text { Mexico, Ecuador, } \\
\text { Egypt, Guatemala, } \\
\text { Morocco, Somalia, } \\
\text { Ghana, Argentina }\end{array}$} \\
\hline & & & & & & $\begin{array}{l}\text { Interviews and } \\
\text { observations }\end{array}$ & \\
\hline & & & & & & & \\
\hline & & & & & & & $\begin{array}{l}\text { Refugee, } \\
\text { undocumented, } \\
\text { immigrant }\end{array}$ \\
\hline \multirow[t]{5}{*}{65.} & \multirow{5}{*}{$\begin{array}{l}\text { Wikberg } 2012 \\
{[106]}\end{array}$} & \multirow{5}{*}{$\begin{array}{l}\text { To describe and } \\
\text { interpret from an } \\
\text { intercultural } \\
\text { perspective, the } \\
\text { perceptions and } \\
\text { experiences of } \\
\text { immigrant new } \\
\text { mothers in maternity } \\
\text { care in Finland }\end{array}$} & \multirow[t]{5}{*}{ Finland } & \multirow[t]{5}{*}{ Maternity care } & \multirow{5}{*}{$\begin{array}{l}\text { Focused } \\
\text { ethnography }\end{array}$} & 17 mothers & \multirow{2}{*}{$\begin{array}{l}\text { Australia, Bosnia, } \\
\text { Burma, Colombia, } \\
\text { Estonia, Hungary, India, } \\
\text { Iraq, Russia, Thailand, } \\
\text { Uganda, Vietnam }\end{array}$} \\
\hline & & & & & & \multirow{4}{*}{$\begin{array}{l}\text { Interviews, } \\
\text { observations, and } \\
\text { documents } \\
\text { (information given to } \\
\text { mothers, newspapers, } \\
\text { websites and informal } \\
\text { notes) }\end{array}$} & \\
\hline & & & & & & & $\begin{array}{l}\text { LOT: < } 1 \text { year to } 10 \\
\text { years }\end{array}$ \\
\hline & & & & & & & $\begin{array}{l}\text { Refugee, immigrant, } \\
\text { family sponsored, } \\
\text { asylum seeker }\end{array}$ \\
\hline & & & & & & & Kurdish \\
\hline 66. & Wikberg 2014 & To illuminate & Finland & Maternity care & Focused & 17 mothers & Australia, Bosnia, \\
\hline & & mothers' experiences & & & & $\begin{array}{l}\text { Interviews, } \\
\text { observations, and }\end{array}$ & Estonia, Hungary, India, \\
\hline
\end{tabular}


Table 3 Description of included literature (Continued)

\begin{tabular}{|c|c|c|c|c|c|c|c|}
\hline \# & $\begin{array}{l}\text { 1st Author } \\
\text { year }\end{array}$ & Objective & $\begin{array}{l}\text { Country } \\
\text { Location }\end{array}$ & $\begin{array}{l}\text { Care, program or } \\
\text { intervention } \\
\text { examined }\end{array}$ & $\begin{array}{l}\text { Design/ } \\
\text { Methodology }^{\text {a }}\end{array}$ & $\begin{array}{l}\text { Sample \& Data } \\
\text { collection methods }\end{array}$ & $\begin{array}{l}\text { Migrant groups } \\
\text { included in the } \\
\text { research }\end{array}$ \\
\hline & & $\begin{array}{l}\text { and perceptions of } \\
\text { caring in maternity }\end{array}$ & & & & $\begin{array}{l}\text { documents } \\
\text { (information given to }\end{array}$ & $\begin{array}{l}\text { Iraq, Russia, Thailand, } \\
\text { Uganda, Vietnam }\end{array}$ \\
\hline & & services in Finland & & & & $\begin{array}{l}\text { mothers, newspapers, } \\
\text { websites and informal } \\
\text { notes) }\end{array}$ & $\begin{array}{l}\text { LOT: < } 1 \text { year to } 10 \\
\text { years }\end{array}$ \\
\hline & & & & & & & $\begin{array}{l}\text { Refugee, immigrant, } \\
\text { family sponsored, } \\
\text { asylum seeker }\end{array}$ \\
\hline & & & & & & & Kurdish \\
\hline \multirow[t]{2}{*}{67.} & \multirow[t]{2}{*}{$\begin{array}{l}\text { Willey } 2018 \\
\text { [108] }\end{array}$} & \multirow{2}{*}{$\begin{array}{l}\text { To explore service } \\
\text { provision for Victorian } \\
\text { regional refugee } \\
\text { families from the } \\
\text { perspective of maternal } \\
\text { and child health nurses }\end{array}$} & \multirow[t]{2}{*}{ Australia } & \multirow[t]{2}{*}{$\begin{array}{l}\text { Maternal and child } \\
\text { primary healthcare }\end{array}$} & \multirow[t]{2}{*}{$\begin{array}{l}\text { Qualitative } \\
\text { descriptive }\end{array}$} & $\begin{array}{l}26 \text { maternal and child } \\
\text { health nurses }\end{array}$ & $\mathrm{N} / \mathrm{A}$ \\
\hline & & & & & & $\begin{array}{l}\text { Focus groups, } \\
\text { questionnaire }\end{array}$ & \\
\hline \multirow[t]{3}{*}{68.} & \multirow[t]{3}{*}{$\begin{array}{l}\text { Wojnar } 2015 \\
{[109]}\end{array}$} & \multirow{3}{*}{$\begin{array}{l}\text { To explore the } \\
\text { perspective of Somali } \\
\text { couples on care and } \\
\text { support received } \\
\text { during the perinatal } \\
\text { period in the United } \\
\text { States }\end{array}$} & \multirow[t]{3}{*}{$\begin{array}{l}\text { United } \\
\text { States }\end{array}$} & \multirow[t]{3}{*}{ Perinatal healthcare } & \multirow[t]{3}{*}{$\begin{array}{l}\text { Descriptive } \\
\text { phenomenology }\end{array}$} & $\begin{array}{l}26 \text { mothers and } 22 \\
\text { fathers }\end{array}$ & Somalia \\
\hline & & & & & & Interviews & LOT: $\leq 5$ years \\
\hline & & & & & & & Refugee \\
\hline \multirow{5}{*}{69.} & \multirow{5}{*}{$\begin{array}{l}\text { Yelland } 2014 \\
\text { [110] }\end{array}$} & \multirow{5}{*}{$\begin{array}{l}\text { To explore the } \\
\text { responsiveness of } \\
\text { health services to the } \\
\text { social and mental } \\
\text { health of Afghan } \\
\text { women and men at } \\
\text { time of having a baby }\end{array}$} & \multirow[t]{5}{*}{ Australia } & \multirow{5}{*}{$\begin{array}{l}\text { Maternity and early } \\
\text { childhood services }\end{array}$} & \multirow{5}{*}{$\begin{array}{l}\text { Qualitative } \\
\text { descriptive }\end{array}$} & \multirow{4}{*}{$\begin{array}{l}16 \text { mothers and } 14 \\
\text { fathers } 34 \text { health } \\
\text { professionals } \\
\text { (midwives, general } \\
\text { practitioners, refugee } \\
\text { health nurses, } \\
\text { maternal and child } \\
\text { health nurses, } \\
\text { obstetricians, } \\
\text { community bicultural } \\
\text { workers and other } \\
\text { healthcare personnel) }\end{array}$} & Afghanistan \\
\hline & & & & & & & $\begin{array}{l}\text { LOT: }<1 \text { year to } \geq 6 \\
\text { years }\end{array}$ \\
\hline & & & & & & & Refugee \\
\hline & & & & & & & $\begin{array}{l}\text { Hazera, Tajik, Pashtu, } \\
\text { Afghan, Sadath }\end{array}$ \\
\hline & & & & & & $\begin{array}{l}\text { Interviews and focus } \\
\text { groups }\end{array}$ & \\
\hline
\end{tabular}

\footnotetext{
${ }^{a}$ For qualitative research in cases where the methodology was not explicitly named or it was unclear, we applied a label based on the description provided. General 'qualitative' exploratory studies were categorized as 'qualitative descriptive'

${ }^{b}$ The description of migrants is based on what was provided in the paper (country of origin, length of time (LOT*) in country, immigration status, and/or ethnicity); Often 'immigrant' is used to generally refer to anyone foreign-born without specifying which immigration statuses are included
}

refugee families with a child aged 0 to 6 years [108]; papers that reported on parents and care-providers' perspectives of peer support group interventions meant to enhance parenting skills, promote child development and reduce isolation among immigrant mothers [22] and refugee mothers/parents [80, 101-103]; and literature that described and/or evaluated specialized earlyeducation programs for migrant and refugee families, or that investigated the experiences of educators in providing these services $[60,73,76,90,98,99]$.

The five remaining papers examined care or programs that spanned the periods of maternity and early-childhood (see Tables 3 and 4). These included: three that examined asylum seeker and refugee mothers' experiences accessing various health and social services during pregnancy and early motherhood $[81,91,110]$; two of these $[91,110]$ also considered the perspectives of healthcare professionals, and one also included the experiences of fathers [110]; one article that inquired on mothers' experiences at a community based perinatal health and social centre which provides medical and psychosocial care to families living in vulnerable contexts from pregnancy up to age five [31]; and another paper that explored the experiences of community workers who provide care and support to refugee and asylum seeker mothers in the perinatal and post-birth periods [54].

The research literature ( $n=66$ studies) primarily reflects parents' views and experiences with care, services and/or programs/interventions; $80 \%$ of studies included parents and $42 \%$ included care-providers (see Table 4). Parents' perspectives are mainly those of mothers' $(n=$ 52 studies); ten studies included fathers $[68,79,85,98$, $101-103,105,109,110]$. The migrant populations studied were mostly from non-western countries; more than 
Table 4 Summary of the literature by publication type, location, focus and populations studied

\begin{tabular}{|c|c|}
\hline Descriptor & $\boldsymbol{N}=69$ papers $\%(n)$ \\
\hline \multicolumn{2}{|l|}{ Publication type } \\
\hline Qualitative research & $82.6 \%(57)$ \\
\hline Quantitative research & $2.9 \%(2)$ \\
\hline Mixed-methods research & $10.1 \%(7)$ \\
\hline Discussion paper & $4.3 \%(3)$ \\
\hline \multicolumn{2}{|l|}{ Location of study/discussion paper } \\
\hline Europe $^{a}$ & $40.6 \%(28)$ \\
\hline United States & $23.2 \%(16)$ \\
\hline Australia and New Zealand & $20.3 \%(14)$ \\
\hline Canada & $15.9 \%(11)$ \\
\hline \multicolumn{2}{|c|}{ Focus of service/care/program/intervention examined } \\
\hline Maternity (prenatal, birth, and postpartum) & $69.6 \%(48)$ \\
\hline Early childhood (post-birth up to age five) & $23.2 \%(16)$ \\
\hline Maternity and early childhood & $7.3 \%(5)$ \\
\hline Populations studied & $N=66$ studies $\%(n)$ \\
\hline Parents & $57.6 \%(38)$ \\
\hline Care-providers & $19.7 \%(13)$ \\
\hline Care-providers and parents & $22.7 \%(15)$ \\
\hline Parents & $N=53$ \\
\hline Mothers & $81.1 \%(43)$ \\
\hline Fathers & $1.9 \%(1)$ \\
\hline Both parents ${ }^{b}$ & $17.0 \%(9)$ \\
\hline Migration source region of parents ${ }^{c}$ & $N=53$ \\
\hline Sub-Saharan Africa & $56.6 \%(30)$ \\
\hline North Africa/Middle East & $41.5 \%(22)$ \\
\hline Latin America & $32.0 \%(17)$ \\
\hline Caribbean & $7.5 \%(4)$ \\
\hline South Asia & $43.4 \%(23)$ \\
\hline South East Asia & $20.8 \%(11)$ \\
\hline East Asia & $17.0 \%(9)$ \\
\hline Eastern Europe/Russia & $24.5 \%(13)$ \\
\hline Western Europe/Australia & $9.4 \%(5)$ \\
\hline Migration status of parents ${ }^{c}$ & $N=53$ \\
\hline Immigrant $^{d}$ & $56.6 \%(30)$ \\
\hline Family & $9.4 \%(5)$ \\
\hline Refugee & $54.7 \%(29)$ \\
\hline Asylum-seeker & $20.8 \%(11)$ \\
\hline Undocumented (non-status migrants) & $17.0 \%(9)$ \\
\hline
\end{tabular}

Includes Austria, Belgium, Finland, France, Germany, Greece, Ireland, Italy, Netherlands, Norway, Portugal, Spain, Sweden and the United Kingdom

${ }^{b}$ One study also included grandparents and other members of their community

${ }^{\mathrm{C}} \mathrm{A}$ study may be counted in more than one category so percentages do not add to $100 \%$

dImmigrant was often used as a general term to describe the foreignborn population and so may have included migrants with other statuses (i.e., other than economic immigrants) half of the studies (57\%) included migrant parents from Sub-Saharan Africa. South Asia, North Africa/Middle East and Latin America were represented in 43, 42 and $32 \%$ of the research respectively (see Table 4 ). The most common source countries were Somalia $(n=11)$, Sudan $(n=11)$, Afghanistan $(n=10)$, India $(n=9)$ and Iraq $(n=$ 8 ), and refugees were represented in over half of the studies (55\%) (see Table 4).

Regarding the quality of the research, for the qualitative studies, generally it was good- responses to the quality criteria were positive for all of the MMAT items across all papers. The majority of these studies were 'qualitative descriptive' $(n=37)$, which was an appropriate approach given most of the studies sought to explore or describe experiences of receiving or providing care. Grounded theory was the methodology used in three studies $[67,69,78]$, however 'theories' were not generated, raising some question about the validity of using this approach. However, overall the findings across the research were well substantiated by the data and most papers provided in-depth interpretations and discussions of the results.

For the two quantitative studies, one was a small, primarily descriptive, pilot project [68]. The sample of parent participants in this study was relatively small, and based on convenience sampling, but did represent approximately $30 \%$ of the target population, which were Somali clients who were receiving care in the obstetrics department where the study was conducted. Details on the number of care-provider participants were not provided. The measurements and analyses were appropriate for the research objectives. The second study, a randomized control trial, only reported preliminary descriptive data about the participants and their participation in the intervention [90]. The methods, nonetheless, were well described, randomization was appropriate, and differences between intervention groups at baseline were explained; one important limitation was that measurement tools were not translated to migrants' languages which may affect the validity of the eventual outcomes of the research.

For the mixed-methods studies, one study provided inadequate details on the methods and was therefore deemed poor quality [75]; the other six were all good quality- there was a clear rationale for using mixedmethods, and the qualitative and quantitative components were sufficiently described and 'mixed' $[60,88,97$, 100-102]. Two strengths across the research, irrespective of the design, were: 1 - the inclusion of minority language migrants as participants; of the 53 studies with migrant parents, 36 accommodated those speaking a language other than the host-country language; and 2- the migrant populations were generally well-described using key indicators (i.e., country of origin, length of time, and migration status). 


\section{Transnationalism and care}

Across the literature, it was evident that many migrant families value their 'ways of belonging' and wish to maintain a transnational identity, especially for their children [21, 31, 49, 67, 69-72, 85, 86, 89, 93-100, 102, $103,105,109]$. A number of papers also showed that cultural, religious and linguistic ties are often acknowledged and addressed in health and social care. Cultural and religious ways of belonging were attended to in various ways. These included: the use of community-based doulas, mostly other mothers from the same communities, who acted as "cultural bridges" and provided information on different cultural birthing customs [47]; hiring of a cleric to conduct religious traditions and prayers in the hospital [21]; the involvement of peers in support/community groups and programs towards enhancing social support and healthy behaviors through the sharing of knowledge [41, 70, 80, 90-92, 101]; the creation and use of culturally-adapted materials including a prenatal training course [75], a psycho-education program [99], a specialized antenatal clinic [100], perinatal health education videos [68], and a mhealth application developed to support a healthy pregnancy and to connect women to services and other women in their ethnic community [48, 49]; and the adaptation or tailoring of care to respond to different cultural and religious needs and expectations, for example having a female care-provider, asking about food preferences and providing culturally appropriate recommendations on prenatal nutrition, baby care, and parenting $[41,53,54,58,61$, $65,78,79,84,86,92-95,97,98,103,106-108,110]$.

Language was also addressed in care using different approaches. This was mostly done by accommodating migrants through the use of interpreters, inter-bicultural/lingual health workers or liaisons and translated materials $[41,46,47,49,53-55,58,61,65,73,76-78$, 84, 86, 90-92, 94, 96, 98, 100, 103-108, 110]. Referring women to community supports where care providers speak migrants' language, was also used to ensure linguistically adapted care [108]. When language could not be accommodated, some care-providers used smiling and having a kind demeanor as a means to make women feel accepted and to show compassion and empathy, regardless of language barriers [59, 104]. Some careproviders made an effort to learn a few words of the migrants' language to create connection and make women feel more comfortable [53]. Speaking more slowly, adjusting their words and using miming or non-verbal expression was also used when language was a challenge [77-79, 86, 91, 106, 107]. In three papers, care-providers actively encouraged families to speak their native language and to pass it on to their children [31, 101, 105]; in one of these, maintaining culture was also promoted [2]. And lastly, accessing care from care-providers who were from the same ethnic community was also a way for migrants to feel respected and understood in their culturally and linguistically 'ways of belonging' [52, 62, 74, 96].

Despite there being evidence that 'ways of belonging' are addressed in care, there were also several papers that showed that care-providers are not sufficiently aware and responsive to cultural, religious and linguistic ties. Migrants reported challenges with communication, including not being able to express themselves or to understand information $[50,51,54,55,59,62,64,66$, $69,70,74,79,81,82,85,86,88,89,91,92,96,97,100$, $102,106,107,109,110]$, care not being culturally appropriate, for example post-birth and infant care practices not being respected or accommodated, and having unmet expectations due to different experiences in their home countries (e.g., anticipating more or less prenatal visits, ultrasounds and tests) [21, 52, 54-56, 61-64, 66, 67, 69-71, 74, 82, 86, 93, 95-97, 100, 102, 105-107, 110]. Similarly, care-providers also encountered difficulties assessing, understanding and responding to needs due to language and cultural barriers $[53,58,60,65,73$, $77,78,84,87,88,91,100,102,104,108]$. Across the literature a need for making care more 'culturally safe' was identified and deemed essential, including having a more diverse and trained (on cultural competency) healthcare workforce, greater use of linguistic/cultural brokers, and more openness to different ways of doing and/or simply acknowledgement, humility/respect and kindness towards difference $[21,47,50,51,53,56,57,59,62,66$, $67,70,72-74,77-79,81,83,84,86-89,91,94,96,98-$ 102, 104, 106-109].

Other forms of transnational ties (i.e., 'ways of being') were mentioned in fewer papers. This included references to families worrying about relatives, including children who remained in the home country $[54,55,59,60$, $67,86,105]$ and families staying connected via phone/ communication technologies and/or visits as a source of social and emotional support, to maintain cultural identity, and/or for assistance with childcare [21, 22, 31, 48, $49,67,69,85,90,95,96,102,105]$. It also included families sending money back home [105], women seeking healthcare in their country to give birth [21], and families using transnational networks to obtain advice and health information- partly due to local care not being adapted [21, 49, 88, 94, 96, 105].

Overall, there was very little evidence showing that care-providers consider and address 'ways of being' in their interventions or care. Some literature showed that care-providers were aware that family separation was causing distress and/or the papers concluded that this issue should be addressed in care $[54,60,86,110]$. In Yelland (2014), two-thirds of women reported being asked by a doctor, midwife or maternal and child health 
nurse about their family members in Australia and back home; one participant expressed that she would have liked to have been asked about her parents [110]. In Rickmeyer et al. (2015), care for a refugee woman participating in an early-childhood program included discussing the relationships that a woman maintained with her family back home (a source of support for her). This intervention was comforting for the woman and also enhanced the rapport with the care-provider who was then able to support the woman to work through her trauma [90]. Another paper mentioned that midwives thought that women may be having children in order to receive more governmental family allowance with the intention to use the money to financially support family members back home, although there was no indication on whether this was discussed or addressed with families [77]. In two other papers (by the same authors), the role of family back home as a source of emotional and social support during pregnancy was recognized and incorporated into a mhealth intervention; the application provided a forum for sharing information and could also be used to involve family members in prenatal care visits via conference calls $[48,49]$.

No studies explicitly examined care-providers' perceptions of transnational ties, however a number of studies suggest that there is a mix of views, including some healthcare professionals having a positive attitude towards different ways of belonging, including a tolerance and/or openness to different cultures, religions and/or identities [53, 58, 66, 78, 84, 95, 104, 106, 107, 110], and others having the view that migrants should fully assimilate and conform to the healthcare system of the new country, such as learning the country's language and not having issue with male care-providers $[53,58,65,66,78$, $84,104]$. In some instances, healthcare professionals reported feeling left-out when providing care across languages $[47,58]$. On the other hand, some migrants felt that their care-providers did not care about them [70] while others reported experiencing stereotyping and discrimination $[55,66,82,102,106,107,109]$. Careproviders also reported witnessing migrants being treated differently due to their cultural, religious and linguistic differences [87, 88, 104].

Regarding migrants' transnational 'ways of being', it remains unknown what care-providers think- none of the literature revealed how care-providers perceive the connections that migrants maintain with their home country, including social, emotional and economic ties and/ or the use of health or other services abroad. However, there is some suggestion that some care-providers are concerned about, and empathize with migrant families that are distressed and worried about their loved ones who have remained in the home country [60]. In this paper, educators working with refugees reported that they found it distressing to hear what families were dealing with and they felt that more support should be provided in this regard. Conversely, there is also a suggestion that some healthcare providers are unsympathetic to migrants' connections to the home country and/or feel that it is not within their scope of practice to inquire about them $[69,88]$.

\section{Discussion}

The results of the review show that transnational ties in terms of 'ways of belonging' are acknowledged and addressed in care provided to migrant families during pregnancy, postpartum and early childhood, although important gaps remain. There is little evidence however, that transnational ties, in terms of 'ways of being', are acknowledged and addressed by care providers. Perceptions of 'ways of belonging' appear to be mixed, with some care-providers being open to and willing to adapt care to accommodate religious, cultural and linguistic differences, while others are not. How care-providers perceive the social, emotional and economic ties and/or the use of services abroad, remains relatively unknown.

Significant attention has been given to cultural competency and cultural safety as approaches to improve the quality of care and to reduce health inequities among migrants, as well as indigenous and other ethnic minority groups [111-113]. Cultural competency generally refers to a set of attitudes and practices towards creating health and social care environments where cultural, religious and linguistic ties are respected and accommodated [114]. Cultural safety involves acknowledging and addressing power differentials in clinical interactions and the broader system and structures that result from social, economic, political, and historical circumstances [112]. Results from the current review show that continued efforts are needed to ensure that pregnancy, postbirth and early childhood care and interventions are adapted and culturally safe for migrants (recommendations and some additional resources are reported in Table 5). Given that many migrant families value maintaining a transnational identity, especially for their children, care should also involve supporting migrants to practice their cultural and religious traditions and to speak their native languages. Although there is evidence that diversity is respected and accommodated, it does not appear to be promoted, and emphasis still tends to be on integration (i.e., addressing settlement issues and encouraging the learning of the host-country language and culture) [39-41, 115, 116]. Maintaining a transnational identity, however, is not counter to integration, both processes may occur simultaneously [12], and preserving cultural, religious and linguistic ties may provide an alternate source of belonging, contribute to social and economic capital and have health benefits for 
Table 5 Recommendations for practice and resources

For supporting Ways of belonging'
Increase the diversity of the healthcare workforce
Offer tailored, community-based programs and services for migrants
Partner with communities and organizations to develop interventions
and programs
Provide cultural competence training to all health and social care
personnel
Use linguistically and culturally-adapted materials
Use trained linguistic, cultural brokers
Reflect on your own culture, beliefs and attitudes about 'others'
Recognize and avoid stereotypes
Attend to power differentials and engage in two-way dialogue
Humbly acknowledge that you are a learner and remain open to
different ways of knowing and doing
Communicate in a value free, respectful tone
Tailor care to the context
Actively counter racism and discrimination
For supporting Ways of being'
Inquire about family members, including children back home
Acknowledge transnational stressors (e.g., economic commitments,
family responsibilities, stressful relationships)
Ask about the use of health and social services abroad
Ask about other health information, support, advice and medicines
coming from back home
Resources: cultural competency and cultural safety
The Office of Minority Health: https://minorityhealth.hhs.gov
The National Center for Cultural Competence: https://nccc.georgetown.
edu
Health Information Translations, Quality health education resources for
diverse populations: https://Healthinfotranslations.org
EthnoMed: https://ethnomed.org
Diversity Rx: www.diversityrx.org
HealthReach, Health information in Many Languages: https://
healthreach.nlm.nih.gov

migrant families [5, 7, 24, 27, 94]. In turn, these positive outcomes may actually reinforce integration.

The review findings are consistent with other research that shows migrant families maintain emotional, social and economic ties with family, and also use health services and receive support from the home country [1]. There is a growing body of research that shows that these transnational ties can have both positive and negative impacts on migrants' lifestyle behaviours, disease management, social well-being, mental health and information and treatment seeking activities [20, 29, 117121]. Moreover, migrants have reported having to reconcile differences between advice received from family members back home and care-providers on health related matters in maternal or child care [105]. Evidently, if care-providers do not consider the transnational stresses (concern for family members, economic strain), sources of support and use of healthcare, they will lack a full understanding of migrants' everyday lives and of the potential impact that these ties may have on migrants' health. This may then affect care-providers' relationships with migrant families and/or the effectiveness of their health and social care interventions. Although the review did not shed much light on how exactly these forms of transnational ties should be addressed in care, it did show that inquiring about family members back home could be used to build rapport $[90,110]$, and that technology could be used to foster the involvement and support of family living far away in maternity care [49]. Without evidence, it is premature to make specific recommendations for care, however, based on the findings, healthcare providers should at minimum, be cognizant of and inquire about migrants' transnational contexts that may affect their health and well-being (see Table 5).

Care-providers' views of migrants also have an impact on care. Othering and discrimination towards migrants in the healthcare system based on culture, language, race and religious differences are known issues and affect access to and quality of services [122-124]. These practices involve implicit biases as well as overt racism and discrimination where migrants are viewed differently and provided sub-optimal care, which are justified through explanations of culturalism and racialization [122]. Through culturalism and racialization migrants' are perceived as inherently inferior due to their worldviews, beliefs and race, and their health and care disparities are seen as being the result of their cultural and religious practices and genetics. In this process migrants are also categorized into differentiated groups and not viewed as individuals, thus further contributing to an 'us' vs. 'them' dichotomy. Having a 'foreign' status, especially being a refugee or not having a legal status, exacerbates this divisiveness and adds to the notion that migrants merit less. Recognizing these biases and harmful ideologies and actively countering racism and discrimination are therefore also key to supporting ways of belonging and creating a care environment that is safe (Table 5). Care-providers can combat racism and discrimination by first being aware of their prejudices and consciously altering their language and behaviours while interacting with migrants $[122,125]$. They may also sensitize and educate others by modelling behaviour, speaking openly about issues of racism and discrimination and condemning unjust treatment of migrants $[122,125]$. Cultural competency training should also be complemented with anti-racism training.

To further develop care approaches and foster a safe care environment, it would be informative to know what care professionals think about transnational 'ways of 
being'- whether they deem economic, social and emotional ties to the home countries as problematic- for example, as a hindrance to integration, or as a factor that contributes to social isolation; and/or whether they view them positively, for example as a source of support and/ or for maintaining cultural and linguistic identity. It would also be useful to know whether they consider these to be within their scope of care. While careproviders may be open to accommodating different cultures and languages, they may be less willing to consider and involve people abroad and/or to extend their care to concerns outside their borders.

The review highlighted research gaps. Future research is needed to determine if and how care providers acknowledge and address transnational 'ways of being' (social, emotional, economic ties and use of health services) in care during pregnancy, postpartum and early childhood. We also need research to learn about what careproviders think and feel regarding migrants' 'ways of being' and also to hear the perspectives of migrants on how they feel these transnational ties should (or should not) be addressed in care. The viewpoints of both mothers and fathers should be examined since these are likely gendered experiences $[119,121]$, and since fathers overall are less represented in research on pregnancy, postpartum and early intervention care.

\section{Limitations and strengths}

The searches yielded a vast amount of records to sort through, therefore it is possible that some literature was missed. We only included papers that focused on health promotion generally and with healthy populations, papers that focused on treatments or one aspect of health promotion, and/or ill or disabled populations, may have included pertinent information regarding transnational ties and care. We also did not include grey literature which may have had relevant information as well. It is worthy to note that no papers specifically inquired about transnational ties and care, so the conclusions drawn in this review are based on the examination of study results that generally captured experiences of care and/or descriptions of interventions and programs. Given this indirect approach and the type of data extracted, it was not possible to draw definitive conclusions regarding the extent to which transnational ties are acknowledged and addressed in care or about the views of healthcare professionals on transnational ties. Despite these limitations, we used a thorough approach to identify, select and analyse papers and we synthesized a broad body of literature representing a range of migrant populations living in a number of different countries. We only used primary sources to ensure we could assess data directly, and we included studies that examined parents' experiences as well as those that examined care-providers' experiences.
As far as we are aware this is the first review to examine transnational ties and health and social care supporting migrant families during pregnancy, postpartum and early childhood.

\section{Conclusion}

To ensure migrants have equitable access to effective, quality care during pregnancy, postpartum and early childhood, further work is needed to raise the critical consciousness of care-providers and to create more culturally safe interventions and care environments. Future research is needed to know whether and how careproviders' take into account transnational 'ways of being', including relationships with children and other family members who remained in the home country, use of health services abroad, and receipt of advice and support from family back home, which may affect their relationships with migrant families and/or the effectiveness of their health and social care interventions. Research is also needed to know the perspectives of care-providers on migrant families' transnational 'ways of being'.

\section{Supplementary information}

Supplementary information accompanies this paper at https://doi.org/10. 1186/s12913-020-05632-5.

Additional file 1. Database search results.

\section{Abbreviations}

ECEC: Early childhood education and care; LOT: Length of time;

MCH: Maternal and child health; MMAT: Mixed Methods Appraisal Tool; N/ A: Not applicable

\section{Acknowledgements}

We would like to acknowledge and thank Assia Mourid, the librarian for allied health sciences at the University of Montreal for her assistance in developing the database search strategies and support in conducting the database searches. We would also like to thank Janice Kim for her support in assessing the quality of the empirical literature.

\section{Authors' contributions}

LM conceived the idea for the review, obtained funding, supervised the literature search, review and selection process, quality-assessed the empirical studies, synthesized and analyzed the literature and drafted the manuscript. VS and NLH developed the database search strategies (with assistance from a librarian), conducted the searches, reviewed and selected the literature, and extracted the data and created the summary tables; VS also aided in the writing of the methods section of the manuscript. SFV provided critical input on the results of the review and revised the manuscript for intellectual content. All authors read and approved the final version of the manuscript.

\section{Funding}

This research, including research assistant support and publication fees, was funded by The Fonds de recherche du Québec - Société et culture (FRQSC), Research Support for New Academics 2018-2019 competition (Application 2019-NP-252729). LM was supported by a research scholar junior 1 award from The Fonds de recherche du Québec-Santé (FRQS), support for early career researchers.

\section{Availability of data and materials}

Data sharing is not applicable to this article since all data are retrievable from the original sources. Articles included in the review are also summarized in Table 3. 


\section{Ethics approval and consent to participate}

Not applicable.

\section{Consent for publication}

Not applicable.

\section{Competing interests}

The authors declare that they have no competing interests.

\section{Author details}

${ }^{1}$ Faculty of Nursing, University of Montreal, Montreal, Canada. ${ }^{2}$ Department of Public Health, University of Copenhagen, Copenhagen, Denmark. ${ }^{3}$ Schoo of Kinesiology and Exercise Science, University of Montreal, Montreal, Canada. ${ }^{4}$ School of Public Health, University of Montreal, Montreal, Canada.

Received: 15 April 2020 Accepted: 6 August 2020

Published online: 24 August 2020

\section{References}

1. Merry L, Pelaez S, Edwards NC. Refugees, asylum-seekers and undocumented migrants and the experience of parenthood: a synthesis of the qualitative literature. Glob Health. 2017;13(1):75

2. Stewart M, Dennis CL, Kariwo M, Kushner KE, Letourneau N, Makumbe $K$ et al. Challenges faced by refugee new parents from Africa in Canada. J Immigr Minor Health. 2015;17(4):1146-56.

3. Betancourt TS, Abdi S, Ito BS, Lilienthal GM, Agalab N, Ellis H. We left one war and came to another: resource loss, acculturative stress, and caregiverchild relationships in Somali refugee families. Cult Divers Ethn Minor Psychol. 2015:21(1):114.

4. Este DC, Tachble A. Fatherhood in the Canadian context: perceptions and experiences of Sudanese refugee men. Sex Roles. 2009:60(7-8):456-66.

5. Ramsden R, Taket A. Social capital and Somali families in Australia. J Int Migr Integr. 2013:14(1):99-117.

6. Osman F, Klingberg-Allvin M, Flacking R, Schön U-K. Parenthood in transition-Somali-born parents' experiences of and needs for parenting support programmes. BMC Int Health Hum Rights. 2016;16(1):7.

7. Carranza ME. Building resilience and resistance against racism and discrimination among Salvadorian female youth in Canada. Child Family Social Work. 2007:12(4):390-8

8. Rasmussen A, Akinsulure-Smith A, Chu T, Keatley E. "911" among west African immigrants in new York City: a qualitative study of parents' disciplinary practices and their perceptions of child welfare authorities. Soc Sci Med. 2012;75(3):516-25

9. Lo M-CM. Cultural capital, motherhood capital, and low-income immigrant mothers' institutional negotiations. Sociol Perspect. 2016;59(3):694-713.

10. Vesely CK. Low-income African and Latina immigrant mothers' selection of early childhood care and education (ECCE): considering the complexity of cultural and structural influences. Early Child Res Q. 2013:28(3):470-86.

11. Levitt $\mathrm{P}$, Jaworsky BN. Transnational migration studies: past developments and future trends. Annu Rev Sociol. 2007:33:129-56.

12. Levitt $\mathrm{P}$, Schiller NG. Conceptualizing simultaneity: a transnational social field perspective on society 1. Int Migr Rev. 2004;38(3):1002-39.

13. Vesely CK, Goodman RD, Ewaida M, Kearney KB. A better life? Immigrant mothers' experiences building economic security. J Fam Econ Iss. 2015;36(4): $514-30$

14. Bonizzoni P. Immigrant working mothers reconciling work and childcare: the experience of Latin American and eastern European women in Milan. Soc Polit. 2014;21(2):194-217.

15. Marcelletti Rocha de Oliveira G. Transnational care constellations: Mexican immigrant mothers and their children in Mexico and in new York City (doctoral dissertation). New York: Columbia University; 2015.

16. Domínguez S, Lubitow A. Transnational ties, poverty, and identity: Latin American immigrant women in public housing. Fam Relat. 2008:57(4):419-30.

17. Abrego $L J$, Menjívar C. Immigrant Latina mothers as targets of legal violence. Int J Sociol Fam. 2011;37(1):9-26

18. Wilding R, Baldassar L. Transnational family-work balance: experiences of Australian migrants caring for ageing parents and young children across distance and borders. J Fam Stud. 2009:15(2):177-87.

19. Haagsman K, Mazzucato V, Dito BB. Transnational families and the subjective well-being of migrant parents: Angolan and Nigerian parents in the Netherlands. Ethn Racial Stud. 2015;38(15):2652-71.
20. Villa-Torres L, Gonzalez-Vazquez T, Fleming PJ, González-González EL, Infante-Xibille C, Chavez R, et al. Transnationalism and health: a systematic literature review on the use of transnationalism in the study of the health practices and behaviors of migrants. Soc Sci Med. 2017:183:70-9.

21. Qureshi R, Pacquiao DF. Ethnographic study of experiences of Pakistani women immigrants with pregnancy, birthing, and postpartum care in the United States and Pakistan. J Transcult Nurs. 2013;24(4):355-62.

22. Beaudet N. Soutenir les mamans immigrantes. Perspective infirmière. 2016; 13(1):52-5.

23. Tingvold L, Middelthon A-L, Allen J, Hauff E. Parents and children only? Acculturation and the influence of extended family members among Vietnamese refugees. Int J Intercult Relat. 2012;36(2):260-70.

24. Machado-Casas M. The politics of organic phylogeny: the art of parenting and surviving as transnational multilingual Latino indigenous immigrants in the US. High Sch J. 2009;92(4):82-99.

25. Jessri M, Farmer AP, Olson K. Exploring middle-eastern mothers' perceptions and experiences of breastfeeding in Canada: an ethnographic study. Maternal Child Nutrition. 2013;9(1):41-56.

26. Urrieta $L \mathrm{Jr}$, Martínez $\mathrm{S}$. Diasporic community knowledge and school absenteeism: Mexican immigrant pueblo Parents' and Grandparents' postcolonial ways of educating. Interventions. 2011;13(2):256-77.

27. Longman C, De Graeve K, Brouckaert T. Mothering as a citizenship practice: an intersectional analysis of 'carework'and 'culturework'in non-normative mother-child identities. Citizsh Stud. 2013;17(3-4):385-99.

28. Bohr Y, Tse C. Satellite babies in transnational families: a study of parents' decision to separate from their infants. Infant Ment Health J. 2009:30(3):265-86.

29. Viruell-Fuentes EA, Schulz AJ. Toward a dynamic conceptualization of social ties and context: implications for understanding immigrant and Latino health. Am J Public Health. 2009;99(12):2167-75.

30. Mwembo L. Congolese immigrant parents' perceptions about their children's education in Philadelphia (doctoral dissertation). Philadelphia: Temple University; 2009

31. Aubé T, Pisanu S, Merry L. La Maison Bleue: strengthening resilience among migrant mothers living in Montreal, Canada. PLoS One. 2019;14(7):e0220107.

32. Sabharwal M, Varma R. Return migration to India: decision-making among academic engineers and scientists. Int Migr. 2016:54(4):177-90.

33. Andrews TJ, Ybarra V, Matthews LL. For the sake of our children: Hispanic immigrant and migrant families' use of folk healing and biomedicine. Med Anthropol Q. 2013;27(3):385-413.

34. Grineski SE. Why parents cross for children's health care: transnational cultural capital in the United States-Mexico border region. Soc Theory Health. 2011:9(3):256-74.

35. Rosemberg M-AS, Boutain DM, Mohammed SA. Transnationalism: a framework for advancing nursing research with contemporary immigrants. ANS Advances in nursing science. 2016;39(1):E19.

36. Merry L, Edwards N. Transnationalism and parenthood in a new country. Int J Migration, Health Social Care. 2019;15(4):294-305.

37. Falicov CJ. Working with transnational immigrants: expanding meanings of family, community, and culture. Fam Process. 2007:46(2):157-71.

38. Stone E, Gomez E, Hotzoglou D, Lipnitsky JY. Transnationalism as a motif in family stories. Fam Process. 2005:44(4):381-98.

39. Gonzalez A, Lord G, Rex-Kiss B, Francois JJ. Parents beyond borders: a social group work curriculum for supporting immigrant parents and building solidarity. Social Work with Groups. 2012;35(1):18-34.

40. Stolzenberg R, Berg G, Maschewsky-Schneider U. Healthy upbringing of children through the empowerment of women in a disadvantaged neighbourhood: evaluation of a peer group project. J Public Health. 2012; 20(2):181-92.

41. Coley SL. New baby in a new country: supporting local immigrant pregnant mothers through 'Moms Matter'. Int J Childbirth Education. 2012;27(2):57-62.

42. Dumas JE, Arriaga XB, Moreland Begle A, Longoria ZN. Child and parental outcomes of a group parenting intervention for Latino families: a pilot study of the CANNE program. Cult Divers Ethn Minor Psychol. 2011;17(1):107.

43. Whittemore R, Knafl K. The integrative review: updated methodology. J Adv Nurs. 2005;52(5):546-53.

44. International Organization for Migration (IOM). Glossary on migration, International migration law series No. 25. Geneva: IOM; 2011.

45. Hong QN, Pluye P, Fàbregues S, Bartlett G, Boardman F, Cargo M, et al. Mixed methods appraisal tool (MMAT), version 2018: registration of copyright (\#1148552), Canadian Intellectual Property Office, Industry Canada 2018. 
46. Akhavan S, Edge D. Foreign-born Women's experiences of communitybased doulas in Sweden-a qualitative study. Health Care Women Int. 2012; 33(9):833-48.

47. Akhavan S, Lundgren I. Midwives' experiences of doula support for immigrant women in Sweden-a qualitative study. Midwifery. 2012;28(1):80-5.

48. AlJaberi H. Social interaction needs and entertainment approaches to pregnancy well-being in mHealth Technology Design for low-Income Transmigrant Women: qualitative Codesign study. JMIR Mhealth Uhealth 2018;6(4):e61.

49. AlJaberi $H$. Developing culturally sensitive mHealth apps for Caribbean immigrant women to use during pregnancy: focus group study. JMIR Human Factors. 2018;5(4):e29.

50. Almeida LM, Caldas JP, Ayres-de-Campos D, Dias S. Assessing maternal healthcare inequities among migrants: a qualitative study. Cadernos de Saude Publica. 2014;30:333-40.

51. Almeida LM, Casanova C, Caldas J, Ayres-de-Campos D, Dias S. Migrant women's perceptions of healthcare during pregnancy and early motherhood: addressing the social determinants of health. J Immigrant Minority Health. 2014;16(4):719-23.

52. Anderson LC. The immigrant experience, child feeding and care: an examination of the determinants of Children's health and nutrition in newcomer families (doctoral dissertation). Toronto: University of Toronto (Canada; 2014

53. Ayers BL, Hawley NL, Purvis RS, Moore SJ, McElfish PA. Providers' perspectives of barriers experienced in maternal health care among Marshallese women. Women Birth. 2018;31(5):e294-301.

54. Balaam MC, Kingdon C, Thomson G, Finlayson K, Downe S. 'We make them feel special': the experiences of voluntary sector workers supporting asylum seeking and refugee women during pregnancy and early motherhood. Midwifery. 2016;34:133-40

55. Barkensjö M, Greenbrook JTV, Rosenlundh J, Ascher H, Elden H. The need for trust and safety inducing encounters: a qualitative exploration of women's experiences of seeking perinatal care when living as undocumented migrants in Sweden. BMC Pregnancy Childbirth. 2018;18(1):217.

56. Barona-Vilar C, Más-Pons R, Fullana-Montoro A, Giner-Monfort J, Grau-Muñoz A, Bisbal-Sanz J. Perceptions and experiences of parenthood and maternal health care among Latin American women living in Spain: a qualitative study. Midwifery. 2013;29(4):332-7.

57. Bircher $\mathrm{H}$. Prenatal care disparities and the migrant farm worker community. MCN Am J Matern Child Nurs. 2009;34(5):303-7.

58. Boerleider AW, Francke AL, van de Reep M, Mannien J, Wiegers TA, Deville W. "Being flexible and creative": a qualitative study on maternity care Assistants' experiences with non-Western immigrant women. PLoS One. 2014;9(3):e91843.

59. Briscoe L, Lavender T. Exploring maternity care for asylum seekers and refugees. Br J Midwifery. 2009;17(1):17-24.

60. Busch J, Bihler LM, Lembcke H, Buchmüller T, Diers K, Leyendecker B. Challenges and solutions perceived by educators in an early childcare program for refugee children. Front Psychol. 2018;9:1621.

61. Carolan M, Cassar L. Antenatal care perceptions of pregnant African women attending maternity services in Melbourne, Australia. Midwifery. 2010;26(2): 189-201.

62. Clark L, Redman RW. Mexican immigrant ant mothers' expectations for children's health services. West J Nurs Res. 2007;29(6):670-90.

63. Coutinho E, Rocha A, Pereira C, Silva A, Duarte J, Parreira V. Experiences of motherhood: unmet expectations of immigrant and native mothers, about the Portuguese health system. Aten Primaria. 2014;46:140-4.

64. Doering K, Patterson J, Griffiths C. Experience of the New Zealand maternity care system by a group of Japanese women in one Centre. NZ Coll Midwives J. 2015;51:5-10.

65. Degni F, Suominen S, Essén B, El Ansari W, Vehviläinen-Julkunen K. Communication and cultural issues in providing reproductive health care to immigrant women: health care providers' experiences in meeting Somali women living in Finland. J Immigr Minor Health. 2012;14(2):330-43.

66. Degni F, Suominen SB, El Ansari W, Vehviläinen-Julkunen K, Essen B. Reproductive and maternity health care services in Finland: perceptions and experiences of Somali-born immigrant women. Ethnicity Health. 2014;19(3): 348-66.

67. Dempsey M, Peeren S. Keeping things under control: exploring migrant eastern European womens' experiences of pregnancy in Ireland. $J$ Reproductive Infant Psychol. 2016;34(4):370-82.
68. DeStephano CC, Flynn PM, Brost BC. Somali prenatal education video use in a United States obstetric clinic: a formative evaluation of acceptability. Patient Educ Couns. 2010;81(1):137-41.

69. Gabai N, Furtos J, Maggi-Perpoint C, Gansel Y, Danet F, Monloubou C, et al. Migrant mothers and perinatal care: a transcultural approach. Devenir. 2013; 25(4):285-307

70. Grewal SK, Bhagat R, Balneaves LG. Perinatal beliefs and practices of immigrant Punjabi women living in Canada. J Obstetric Gynecologic Neonatal Nursing. 2008;37(3):290-300

71. Higginbottom GMA, Safipour J, Mumtaz Z, Chiu Y, Paton P, Pillay J. "I have to do what I believe": Sudanese women's beliefs and resistance to hegemonic practices at home and during experiences of maternity care in Canada. BMC Pregnancy Childbirth. 2013;13(1):51.

72. Hill N, Hunt E, Hyrkäs K. Somali immigrant Women's health care experiences and beliefs regarding pregnancy and birth in the United States. J Transcult Nurs. 2012;23(1):72-81

73. Hurley JJ, Warren RA, Habalow RD, Weber LE, Tousignant SR. Early childhood special education in a refugee resettlement community: challenges and innovative practices. Early Child Dev Care. 2014;184(1):50-62

74. Iliadi P. Refugee women in Greece: a qualitative study of their attitudes and experience in antenatal care. Health Sci J. 2008;2(3):173-80.

75. Karl-Trummer U, Krajic K, Novak-Zezula S, Pelikan J. Prenatal courses as health promotion intervention for migrant/ethnic minority women: high efforts and good results, but low attendance. Diversity Health Social Care. 2006;3(1):55-8.

76. Lebiger-Vogel J, Rickmeyer C, Fritzemeyer K, Meurs P, Benzel S, Mazur L, et al. FIRST STEPS: psychoanalytically based prevention projects for migrant and refugee families in Belgium and Germany. Int J Appl Psychoanal Stud. 2019;17:22-38.

77. Lyberg A, Viken B, Haruna M, Severinsson E. Diversity and challenges in the management of maternity care for migrant women. J Nurs Manag. 2012; 20(2):287-95.

78. Lyons SM, O'Keeffe FM, Clarke AT, Staines A. Cultural diversity in the Dublin maternity services: the experiences of maternity service providers when caring for ethnic minority women. Ethn Health. 2008;13(3):261-76.

79. Mangrio E, Persson K. Immigrant parents' experience with the Swedish child health care system: a qualitative study. BMC Fam Pract. 2017;18(1):32.

80. McLaughlin B. Experiences of parenting among Burmese refugee mothers: social support in a playgroup (honors thesis). Perth: Edith Cowan University; 2012.

81. Merry LA, Gagnon AJ, Kalim N, Bouris SS. Refugee claimant women and barriers to health and social services post-birth. Can J Public Health. 2011; 102(4):286-90.

82. Mukasa B. Maternal and child health access disparities among recent African immigrants in the United States (doctoral dissertation). Minneapolis: Walden University; 2016.

83. Nabb J. Pregnant asylum-seekers: perceptions of maternity service provision. Evidence-Based Midwifery. 2006;4(3):89-96.

84. $\mathrm{Ng} \mathrm{C}$, Newbold KB. Health care providers' perspectives on the provision of prenatal care to immigrants. Culture Health Sexuality. 2011;13(05):561-74.

85. Ny P, Plantin L, Dejin-Karlsson E, Dykes A-K. The experience of middle eastern men living in Sweden of maternal and child health care and fatherhood: focus-group discussions and content analysis. Midwifery. 2006; 24(3):281-90.

86. Owens C, Dandy J, Hancock P. Perceptions of pregnancy experiences when using a community-based antenatal service: a qualitative study of refugee and migrant women in Perth, Western Australia. Women Birth. 2016;29(2): 128-37.

87. Peláez S, Hendricks KN, Merry LA, Gagnon AJ. Challenges newly-arrived migrant women in Montreal face when needing maternity care: health care professionals' perspectives. Glob Health. 2017;13(1):5.

88. Phillimore J. Migrant maternity in an era of superdiversity: new migrants' access to, and experience of, antenatal care in the west midlands, UK. Soc Sci Med. 2016;148:152-9.

89. Renzaho A, Oldroyd J. Closing the gap in maternal and child health: a qualitative study examining health needs of migrant mothers in Dandenong, Victoria, Australia. Matern Child Health J. 2014;18(6):1391-402.

90. Rickmeyer C, Lebiger-Vogel J, Busse A, Fritzemeyer K, Burkhardt-Mußmann C, Leuzinger-Bohleber M. Early motherhood in migration: a FIRST report from FIRST STEPS-an integration project for infants with an immigrant background. J Pregnancy Child Health. 2015;2:147. 
91. Riggs E, Davis E, Gibbs L, Block K, Szwarc J, Casey S, et al. Accessing maternal and child health services in Melbourne, Australia: reflections from refugee families and service providers. BMC Health Serv Res. 2012;12(1):117.

92. Riggs E, Muyeen S, Brown S, Dawson W, Petschel P, Tardiff W, et al. Cultural safety and belonging for refugee background women attending group pregnancy care: an Australian qualitative study. Birth. 2017:44(2):145-52.

93. Russo A, Lewis B, Joyce A, Crockett B, Luchters S. A qualitative exploration of the emotional wellbeing and support needs of new mothers from Afghanistan living in Melbourne, Australia. BMC Pregnancy Childbirth. 2015; 15(1):197.

94. Sanchez S, Kasselstrand I. "Provide all women a voice:" a qualitative study of first-generation Mexican immigrant Women's experiences with midwives in the United States. Women's Reproductive Health. 2017;4(3):169-84.

95. Schmiedigen A. The experience of Brazilian women entering motherhood in the United States (doctoral dissertation). Newton: Massachusetts School of Professional Psychology; 2013.

96. Seo YJ, Cheah CSL, Özdemir SB, Hart CH, Leung CYY, Sun S. The mediating role of korean immigrant mothers' psychological well-being in the associations between social support and authoritarian parenting style. J Child Fam Stud. 2017;27(3):979-89.

97. Shafiei T, Small R, McLachlan H. Women's views and experiences of maternity care: a study of immigrant afghan women in Melbourne, Australia. Midwifery. 2012;28(2):198-203.

98. Signorelli RG, Coello M, Momartin S. Change and recovery: culturally appropriate early childhood Programmes with refugee families and communities. Child Aust. 2015;40(3):195-204.

99. Signorelli RG, Gluckman N, Hassan N, Coello M, Momartin S. Relationship building, collaboration and flexible service delivery: the path to engagement of refugee families and communities in early childhood trauma recovery services. J Children Australia. 2017;42(3):142-58.

100. Stapleton H, Murphy R, Correa-Velez I, Steel M, Kildea S. Women from refugee backgrounds and their experiences of attending a specialist antenatal clinic. Narratives from an Australian setting. Women Birth. 2013; 26(4):260-6.

101. Stewart M, Makwarimba E, Letourneau NL, Kushner KE, Spitzer DL, Dennis $\mathrm{CL}$, et al. Impacts of a support intervention for Zimbabwean and Sudanese refugee parents: "I am not alone". Can J Nurs Res. 2015;47(4):113-40.

102. Stewart M, Kushner KE, Dennis C, Kariwo M, Letourneau N, Makumbe K, et al. Social support needs of Sudanese and Zimbabwean refugee new parents in Canada. Int J Migration Health Social Care. 2017;13(2):234-52.

103. Stewart M, Spitzer DL, Kushner KE, Shizha E, Letourneau N, Makwarimba E, et al. Supporting refugee parents of young children: "knowing you're not alone". Int J Migration Health Social Care. 2018;14(1):15-29.

104. Tobin CL, Murphy-Lawless J. Irish midwives' experiences of providing maternity care to non-lrish women seeking asylum. Int J Women's Health 2014;6:159-69.

105. Vesely CK. Learning how to navigate U.S. society with young children: experiences of immigrant mothers utilizing early childhood care and education (doctoral dissertation). College Park: University of Maryland; 2011.

106. Wikberg A, Eriksson K, Bondas T. Intercultural caring from the perspectives of immigrant new mothers. J Obstetric Gynecol Neonatal Nursing. 2012; 41(5):638-49.

107. Wikberg A, Eriksson K, Bondas T. Immigrant new mothers in Finnish maternity care: an ethnographic study of caring. Int J Childbirth. 2014;4(2): 86-102.

108. Willey SM, Cant RP, Williams A, McIntyre M. Maternal and child health nurses work with refugee families: perspectives from regional Victoria, Australia. J Clin Nurs. 2018;27(17-18):3387-96.

109. Wojnar DM. Perinatal experiences of Somali couples in the United States. J Obstet Gynecol Neonatal Nurs. 2015;44(3):358-69.

110. Yelland J, Riggs E, Wahidi S, Fouladi F, Casey S, Szwarc J, et al. How do Australian maternity and early childhood health services identify and respond to the settlement experience and social context of refugee background families? BMC Pregnancy Childbirth. 2014;14(1):348.

111. Renzaho A, Romios P, Crock C, Sønderlund A. The effectiveness of cultural competence programs in ethnic minority patient-centered health care-a systematic review of the literature. Int J Qual Health Care. 2013;25(3):261-9.

112. Curtis E, Jones R, Tipene-Leach D, Walker C, Loring B, Paine S-J, et al. Why cultural safety rather than cultural competency is required to achieve health equity: a literature review and recommended definition. Int J Equity Health. 2019;18(1):174
113. Browne AJ, Varcoe C, Ford-Gilboe M, Wathen CN. EQUIP healthcare: an overview of a multi-component intervention to enhance equity-oriented care in primary health care settings. Int J Equity Health. 2015;14(1):152.

114. Saha S, Beach MC, Cooper LA. Patient centeredness, cultural competence and healthcare quality. J Natl Med Assoc. 2008;100(11):1275-85.

115. Segal UA, Mayadas NS. Assessment of issues facing immigrant and refugee families. Child Welfare. 2005;84(5):563-83.

116. Critelli FM. Parenting in a new land: specialized services for immigrant and refugee families in the USA. J Int Migr Integr. 2015;16(4):871-90.

117. Sanon M-A, Spigner C, McCullagh MC. Transnationalism and hypertension self-management among Haitian immigrants. J Transcult Nurs. 2016;27(2): 147-56.

118. Alcántara C, Molina KM, Kawachi I. Transnational, social, and neighborhood ties and smoking among Latino immigrants: does gender matter? Am J Public Health. 2015;105(4):741-9.

119. Afulani PA, Torres JM, Sudhinaraset M, Asunka J. Transnational ties and the health of sub-Saharan African migrants: the moderating role of gender and family separation. Soc Sci Med. 2016;168:63-71.

120. Alcántara C, Chen C-N, Alegría M. Transnational ties and past-year major depressive episodes among Latino immigrants. Cult Divers Ethn Minor Psychol. 2015;21(3):486.

121. Pannetier J, Lert F, Roustide MJ, Du Loû AD. Mental health of sub-Saharan African migrants: the gendered role of migration paths and transnational ties. SSM-Population Health. 2017;3:549-57.

122. Johnson JL, Bottorff JL, Browne AJ, Grewal S, Hilton BA, Clarke H. Othering and being Othered in the context of health care services. Health Commun. 2004;16(2):255-71.

123. Wahoush EO. Equitable health-care access: the experiences of refugee and refugee claimant mothers with an ill preschooler. Can J Nurs Res. 2009;41(3): 186-206.

124. Pollock G, Newbold KB, Lafrenière G, Edge S. Discrimination in the Doctor's office. Crit Soc Work. 2012;13(2):61-79.

125. Browne AJ, Varcoe CM, Wong ST, Smye VL, Lavoie J, Littlejohn D, et al. Closing the health equity gap: evidence-based strategies for primary health care organizations. Int J Equity Health. 2012;11(1):1-5.

\section{Publisher's Note}

Springer Nature remains neutral with regard to jurisdictional claims in published maps and institutional affiliations.

Ready to submit your research? Choose BMC and benefit from

- fast, convenient online submission

- thorough peer review by experienced researchers in your field

- rapid publication on acceptance

- support for research data, including large and complex data types

- gold Open Access which fosters wider collaboration and increased citations

- maximum visibility for your research: over $100 \mathrm{M}$ website views per year

At $\mathrm{BMC}$, research is always in progress.

Learn more biomedcentral.com/submissions 